\title{
Algorithmic Game Theory
}

Edited by

Noam Nisan, Tim Roughgarden, Éva Tardos, and Vijay Vazirani 

Contents

1 Introduction to Mechanism Design (for Computer Scientists) N. Nisan

page 4 


\title{
Introduction to Mechanism Design (for computer Scientists)
}

\author{
Noam Nisan
}

\begin{abstract}
We give an introduction to the micro-economic field of Mechanism Design slightly biased towards a computer-scientist's point of view.
\end{abstract}

\subsection{Introduction}

Mechanism Design is a sub-field of economic theory that is rather unique within economics in having an engineering perspective. It is interested in designing economic mechanisms, just like computer scientists are interested in designing algorithms, protocols, or systems. It is best to view the goals of the designed mechanisms in the very abstract terms of social choice. A social choice is simply an aggregation of the preferences of the different participants towards a single joint decision. Mechanism Design attempts implementing desired social choices in a strategic setting - assuming that the different members of society each act rationally in a game theoretic sense. Such strategic design is necessary since usually the preferences of the participants are private.

This high-level abstraction of aggregation of preferences may be seen as a common generalization of a multitude of scenarios in economics as well as in other social settings such as political science. Here are some basic classic examples:

- Elections: In political elections each voter has his own preferences between the different candidates, and the outcome of the elections is a single social choice.

- Markets: Classical economic theory usually assumes the existence and functioning of a "perfect market". In reality, of course, we only have interactions between people, governed by some protocols. Each participant 
in such an interaction has his own preferences, but the outcome is a single social choice: the re-allocation of goods and money.

- Auctions: Generally speaking, the more buyers and sellers there are in a market, the more the situation becomes close to the perfect market scenario. An extreme opposite case is where there is only a single seller an auction. The auction rules define the social choice: the identity of the winner.

- Government Policy: Governments routinely have to make decisions that affect a multitude of people in different ways: Should a certain bridge be built? How much pollution should we allow? How should we regulate some sector? Clearly each citizen has a different set of preferences but a single social choice is made by the government.

As the influence of the Internet grew, it became clear that many scenarios happening there can also be viewed as instances of social choice in strategic settings. The main new ingredient found in the Internet is that it is owned and operated by different parties with different goals and preferences. These preferences, and the behavior they induce, must then be taken into account by every protocol in such an environment. The protocol should thus be viewed as taking the preferences of the different participants and aggregating them into a social choice: the outcome of the run of the protocol.

Conceptually, one can look at two different types of motivations, those that use economics to solve computer science issues and those that use computer science to solve economic issues:

- Economics for CS: Consider your favorite algorithmic challenge in a computer network environment: routing of messages, scheduling of tasks, allocation of memory, etc. When running in an environment with multiple owners of resources or requests, this algorithm must take into account the different preferences of the different owners. The algorithm should function well assuming strategic selfish behavior of each participant. Thus we desire a Mechanism Design approach for a multitude of algorithmic challenges - leading to a field that has been termed Algorithmic Mechanism Design.

- CS for Economics: Consider your favorite economic interaction: some type of market, an auction, a supply chain, etc. As the Internet becomes ubiquitous, this interaction will often be implemented over some computerized platform. Such an implementation enables unprecedented sophistication and complexity, handled by hyper-rationally designed software. Designing these is often termed Electronic Market Design. 
Thus, both Algorithmic Mechanism Design and Electronic Market Design can be based upon the field of Mechanism Design applied in complex algorithmic settings.

This chapter provides an introduction to classical Mechanism Design, intended for computer scientists. While the presentation is not very different from the standard economic approach, it is somewhat biased towards a worst-case (non-Bayesian) point of view common in computer science.

Section 1.2 starts with the general formulation of the social choice problem, points out the basic difficulties formulated by Arrow's famous impossibility results, and deduces the impossibility of a general strategic treatment, i.e. of Mechanism Design in the general setting. Section 1.3 then considers the important special case where "money" exists, and describes a very general positive result, the incentive-compatible Vickrey-Clarke-Grove mechanism. Section 1.4 puts everything in a wider formal context of implementation in dominant strategies. Section 1.5 provides several characterizations of dominant strategy mechanisms. All the sections up to this point have considered dominant-strategies, but the prevailing economic point of view is a Bayesian one that assumes a priori known distributions over private information. Section 1.6 introduces this setting and the notion of BayesianNash equilibrium that fits it. All the treatment in this chapter is in the very basic "private value" model, and section 1.7 shortly points out several extensions to the model. Finally, section 1.8 provides bibliographic notes and references.

\subsection{Social Choice}

This section starts with the general social choice problem and continues with the strategic approach to it. The main message conveyed is that there are unavoidable underlying difficulties. We phrase things in the commonly used terms of political elections, but the reader should keep in mind that the issues are abstract and apply to general social choice.

\subsubsection{Condorcet's paradox}

Consider an election with two candidates, where each voter has a preference for one of them. If society needs to jointly choose one of the candidates, intuitively it is clear that taking a majority vote would be a good idea. But what happens if there are three candidates? In 1785, The Marquis de Condorcet pointed out that the natural application of majority is problematic: 
consider three candidates: $a, b$, and $c$, and three voters with the following preferences:

(i) $a \succ_{1} b \succ_{1} c$

(ii) $b \succ_{2} c \succ_{2} a$

(iii) $c \succ_{3} a \succ_{3} b$

(The notation $a \succ_{i} b$ means that voter $i$ prefers candidate $a$ to candidate $b$.) Now, notice that a majority of voters (1 and 3) prefer candidate $a$ to candidate $b$. Similarly, a majority ( 1 and 2$)$ prefers $b$ to $c$, and, finally, a majority (2 and 3) prefers $c$ to $a$. The joint majority choice is thus $a \succ b \succ$ $c \succ a$ which not consistent. In particular for any candidate that is jointly chosen, there will be a majority of voters who would want to change the chosen outcome.

This immediately tells us that in general a social choice cannot be taken simply by the natural system of taking a majority vote. Whenever there are more than two alternatives we must design some more complex "voting method" to undertake a social choice.

\subsubsection{Voting Methods}

A large number of different voting methods - ways of determining the outcome of such multi-candidate elections - have been suggested. Two of the simpler ones are plurality (the candidate that was placed first by the largest number of voters wins) and Borda count (each candidate among the $n$ candidates gets $n-i$ points for every voter who ranked him in place $i$, and the candidate with most points wins). Each of the suggested voting methods has some "nice" properties but also some problematic ones.

One of the main difficulties encountered by voting methods is that they may encourage strategic voting. Suppose that a certain voter's preferences are $a \succ_{i} b \succ_{i} c$, but he knows that candidate $a$ will not win (as other voters hate him). Such a voter may be motivated to strategically vote for $b$ instead of $a$, so that $b$ is chosen which he prefers to $c$. Such strategic voting is problematic as it is not transparent, depends closely on the votes of the other voters, and the interaction of many strategic voters is complex. The main result of this section is the Gibbard-Satterthwaite theorem that states that this strategic vulnerability is unavoidable. We will prove the theorem as a corollary of Arrow's impossibility theorem that highlights the general impossibility of designing voting methods with certain natural good desired properties.

Formally, we will consider a set of alternatives $A$ (the candidates) and 
a set of $n$ voters $I$. Let us denote by $L$ the set of linear orders on $A$ ( $L$ is isomorphic to the set of permutations on $A$ ). Thus for every $\prec \in L, \prec$ is a total order on $A$ (anti-symmetric and transitive). The preferences of each voter $i$ are formally given by $\succ_{i} \in L$, where $a \succ_{i} b$ means that $i$ prefers alternative $a$ to alternative $b$.

Definition 1.1 - A function $F: L^{n} \rightarrow L$ is called a social welfare function. - A function $f: L^{n} \rightarrow A$ is called a social choice function.

Thus a social welfare function aggregates the preferences of all voters into a common preference, i.e. into a total social order on the candidates, while a social choice function aggregates the preferences of all voters into a social choice of a single candidate. Arrow's theorem states that social welfare functions with "nice" properties must be trivial in a certain sense.

\subsubsection{Arrow's Theorem}

Here are some natural properties desired from a social welfare function.

Definition 1.2 - A social welfare function $F$ satisfies unanimity if for every $\prec \in L, F(\prec, \ldots, \prec)=\prec$. I.e. if all voters have identical preferences then the social preference is the same.

- Voter $i$ is a dictator in social welfare function $F$ if for all $\prec_{1} \ldots \prec_{n} \in L$, $F\left(\prec_{1} \ldots \prec_{n}\right)=\prec_{i}$. The social preference in a dictatorship is simply that of the dictator, ignoring all other voters. $F$ is not a dictatorship if no $i$ is a dictator in it.

- A social welfare function satisfies independence of irrelevant alternatives if the social preference between any two alternatives $a$ and $b$ only depends on the voters' preferences between $a$ and $b$. Formally, for every $a, b \in A$ and every $\prec_{1} \ldots \prec_{n}, \prec_{1}^{\prime} \ldots \prec_{n}^{\prime} \in L$, if we denote $\prec=F\left(\prec_{1} \ldots \prec_{n}\right)$ and $\prec^{\prime}=$ $F\left(\prec_{1}^{\prime} \ldots \prec_{n}^{\prime}\right)$ then $a \prec_{i} b \Leftrightarrow a \prec_{i}^{\prime} b$ for all $i$ implies that $a \prec b \Leftrightarrow a \prec^{\prime} b$.

The first two conditions are quite simple to understand and we would certainly want any good voting method to satisfy the unanimity condition and not to be a dictatorship. The third condition is trickier. Intuitively, indeed, independence of irrelevant alternatives seems quite natural: why should my preferences about $c$ have anything to do with the social ranking of $a$ and $b$ ? More careful inspection will reveal that this condition in some sense captures some consistency property of the voting system. As we will see, lack of such consistency enables strategic manipulation. 
Theorem 1.3 (Arrow): Every social welfare function over a set of more than 2 candidates $(|A| \geq 3)$ that satisfies unanimity and independence of irrelevant alternatives is a dictatorship.

Over the years a large number of proofs have been found for Arrow's theorem. Here is a short one.

Proof For the rest of the proof, fix $F$ that satisfies unanimity and independence of irrelevant alternatives. We start with a claim showing that the same social ranking rule is taken within any pair of alternatives.

Claim (pairwise neutrality): Let $\succ_{1} \ldots \succ_{n}$ and $\succ_{1}^{\prime} \ldots \succ_{n}^{\prime}$ be two player profiles such that for every player $i, a \succ_{i} b \Leftrightarrow c \succ_{i}^{\prime} d$. Then $a \succ b \Leftrightarrow c \succ^{\prime} d$, where $\succ=F\left(\succ_{1} \ldots \succ_{n}\right)$ and $\succ^{\prime}=F\left(\succ_{1}^{\prime} \ldots \succ_{n}^{\prime}\right)$.

By renaming we can assume without loss of generality that $a \succ b$ and that $c \neq b$. Now we merge each $\succ_{i}$ and $\succ_{i}^{\prime}$ into a single preference $\succ_{i}$ by putting $c$ just above $a$ (unless $c=a$ ) and $d$ just below $b$ (unless $d=b$ ) and preserving the internal order within each of the pairs $(a, b)$ and $(c, d)$. Now using unanimity we have that $c \succ a$ and $b \succ d$, and by transitivity $c \succ d$. This concludes the proof of the claim.

We now continue with the proof of the theorem. Take any $a \neq b \in A$, and for every $0 \leq i \leq n$ define a preference profile $\pi^{i}$ in which exactly the first $i$ players rank $a$ above $b$, i.e. in $\pi^{i}, a \succ_{j} b \Leftrightarrow j \leq i$ (the exact ranking of the other alternatives does not matter). By unanimity, in $F\left(\pi^{0}\right)$, we have $b \succ a$, while in $F\left(\pi^{n}\right)$ we have $a \succ b$. By looking at $\pi^{0}, \pi^{1}, \ldots, \pi^{n}$, at some point the ranking between $a$ and $b$ flips, so for some $i^{*}$ we have that in $F\left(\pi^{i^{*}-1}\right)$, $b \succ a$, while in $F\left(\pi^{i^{*}}\right), a \succ b$. We conclude the proof by showing that $i^{*}$ is a dictator.

Claim: Take any $c \neq d \in A$. If $c \succ_{i^{*}} d$ then $c \succ d$ where $\succ=F\left(\succ_{1} \ldots \succ_{n}\right)$.

Take some alternative $e$ which is different from $c$ and $d$. For $i<i^{*}$ move $e$ to the top in $\succ_{i}$, for $i>i^{*}$ move $e$ to the bottom in $\succ_{i}$, and for $i^{*}$ move $e$ so that $c \succ_{i^{*}} e \succ_{i^{*}} d$ - using independence of irrelevant alternatives we have not changed the social ranking between $c$ and $d$. Now notice that players' preferences for the ordered pair $(c, e)$ are identical to their preferences for $(a, b)$ in $\pi^{i^{*}}$, but the preferences for $(e, d)$ are identical to the preferences for $(a, b)$ in $\pi^{i^{*}-1}$ and thus using the pairwise neutrality claim, socially $c \succ e$ and $e \succ d$, and thus by transitivity $c \succ d$. 


\subsubsection{The Gibbard-Satterthwaite theorem}

It turns out that Arrow's theorem has devastating strategic implications. We will study this issue in the context of social choice functions (rather than social welfare functions as we have considered until now). Let us start by defining strategic manipulations.

Definition 1.4 A social choice function $f$ can be strategically manipulated by voter $i$ if for some $\prec_{1} \ldots \prec_{n} \in L$ and some $\prec_{i}^{\prime} \in L$ we have that $a \prec_{i} a^{\prime}$ where $a=f\left(\prec_{1} \ldots \prec_{i} \ldots \prec_{n}\right)$ and $a^{\prime}=f\left(\prec_{1} \ldots \prec_{i}^{\prime} \ldots \prec_{n}\right)$. I.e. voter $i$ that prefers $a^{\prime}$ to $a$ can ensure that $a^{\prime}$ gets socially chosen rather than $a$ by strategically mis-representing his preferences to be $\prec_{i}^{\prime}$ rather than $\prec_{i}$. $f$ is called incentive compatible if it cannot be manipulated.

The following is a more combinatorial point of view of the same notion:

Definition 1.5 A social choice function $f$ is monotone if $f\left(\prec_{1} \ldots \prec_{i} \ldots \prec_{n}\right.$ )$=a \neq a^{\prime}=f\left(\prec_{1} \ldots \prec_{i}^{\prime} \ldots \prec_{n}\right)$ implies that $a^{\prime} \prec_{i} a$ and $a \prec_{i}^{\prime} a^{\prime}$. I.e., if the social choice changed from $a$ to $a^{\prime}$ when a single voter $i$ changed his vote from $\prec_{i}$ to $\prec_{i}^{\prime}$ then it must be because he switched his preference between $a$ and $a^{\prime}$.

Proposition 1.6 A social choice function is incentive compatible if and only if it is monotone.

Proof Take $\prec_{1} \ldots \prec_{i-1}, \prec_{i+1} \ldots \prec_{n}$ out of the quantification. Now, logically, "NOT monotone between $\prec_{i}$ and $\prec_{i}^{\prime \prime}$ " is equivalent to "A voter with preference $\prec$ can strategically manipulate $f$ by declaring $\prec$ " OR "A voter with preference $\prec^{\prime}$ can strategically manipulate $f$ by declaring $\prec$ ".

The obvious example of an incentive compatible social choice function over two alternatives is taking the majority vote between them. The main point of this section is, however, that when the number of alternatives is larger than two, only trivial social choice functions are incentive compatible.

Definition 1.7 Voter $i$ is a dictator in social choice function $f$ if for all $\prec_{1} \ldots \prec_{n} \in L, \forall b \neq a, a \succ_{i} b \Rightarrow f\left(\prec_{1} \ldots \prec_{n}\right)=a . f$ is called a dictatorship if some $i$ is a dictator in it.

Theorem 1.8 (Gibbard-Satterthwaite) Let $f$ be an incentive compatible social choice function onto $A$, where $|A| \geq 3$, then $f$ is a dictatorship. 
Note the requirement that $f$ is onto, as otherwise the bound on the size of $A$ has no bite. To derive the theorem as a corollary of Arrow's theorem we will construct a social welfare function $F$ from the social choice function $f$. The idea is that in order to decide whether $a \prec b$, we will "move" $a$ and $b$ to the top of all voters' preferences, and then see whether $f$ chooses $a$ or b. Formally:

Definition $1.9 \bullet$ Notation: Let $S \subset A$ and $\prec \in L$. Denote by $\prec^{S}$ the order obtained by moving all alternatives in $S$ to the top in $\prec$. Formally, for $a, b \in S, a \prec^{S} b \Leftrightarrow a \prec b$; for $a, b \notin S$, also $a \prec^{S} b \Leftrightarrow a \prec b$; but for $a \notin S$ and $b \in S, a \prec^{S} b$.

- The social welfare function $F$ that extends the social choice function $f$ is defined by $F\left(\prec_{1} \ldots \prec_{n}\right)=\prec$, where $a \prec b$ iff $f\left(\prec_{1}^{\{a, b\}} \ldots \prec_{n}^{\{a, b\}}\right)=b$.

We first have to show that $F$ is indeed a social choice function, i.e. that it is anti-symmetric and transitive.

Lemma 1.10 If $f$ is an incentive compatible social choice function onto $A$ then the extension $F$ is a social welfare function.

To conclude the proof of the theorem as a corollary of Arrow's it then suffices to show:

Lemma 1.11 If $f$ is an incentive compatible social choice function onto $A$ which is not a dictatorship then the extension $F$ satisfies unanimity and independence of irrelevant alternatives and is not a dictatorship.

Proof (of Lemmas 1.10 and 1.11) We start with a general claim which holds under the conditions on $f$ :

Claim: For any $\prec_{1} \ldots \prec_{n}$ and any $S, f\left(\prec_{1}^{S} \ldots \prec_{n}^{S}\right) \in S$.

Take some $a \in S$ and since $f$ is onto, for some $\prec_{1}^{\prime} \ldots \prec_{n}^{\prime}, f\left(\prec_{1}^{\prime} \ldots \prec_{n}^{\prime}\right)=a$. Now, sequentially, for $i=1 \ldots n$, change $\prec_{i}^{\prime}$ to $\prec_{i}^{S}$. We claim that at no point during this sequence of changes will $f$ output any outcome $b \notin S$. At every stage this is simply due to monotonicity since $b \prec_{i}^{S} a^{\prime}$ for $a^{\prime} \in S$ being the previous outcome. This concludes the proof of the claim.

We can now prove all properties needed for the two lemmas:

- Anti-symmetry is implied by the claim since $f\left(\prec_{1}^{\{a, b\}} \ldots \prec_{n}^{\{a, b\}}\right) \in$ $\{a, b\}$. 
- Transitivity: assume for contradiction that $a \prec b \prec c \prec a$ (where $\prec=$ $\left.F\left(\prec_{1} \ldots \prec_{n}\right)\right)$. Take $S=\{a, b, c\}$ and using the claim assume without loss of generality that $f\left(\prec_{1}^{S} \ldots \prec_{n}^{S}\right)=a$. Sequentially changing $\prec_{i}^{S}$ to $\prec_{i}^{\{a, b\}}$ for each $i$, monotonicity of $f$ implies that also $f\left(\prec_{1}^{\{a, b\}} \ldots \prec_{n}^{\{a, b\}}\right.$ ) $=a$, and thus $a \succ b$.

- Unanimity: If for all $i, b \prec_{i} a$, then $\left(\prec_{i}^{\{a, b\}}\right)^{\{a\}}=\prec_{i}^{\{a, b\}}$ and thus by the claim $f\left(\prec_{1}^{\{a, b\}} \ldots \prec_{n}^{\{a, b\}}\right)=a$.

- Independence of irrelevant alternatives: If for all $i, b \prec_{i} a \Leftrightarrow b \prec_{i}^{\prime}$ $a$, then $f\left(\prec_{1}^{\{a, b\}} \ldots \prec_{n}^{\{a, b\}}\right)=f\left(\prec_{1}^{\prime\{a, b\}} \ldots \prec_{n}^{\prime\{a, b\}}\right)$ since when we, sequentially for all $i$, flip $\prec_{i}^{\{a, b\}}$ into $\prec_{i}^{\prime\{a, b\}}$, the outcome does not change due to monotonicity and the claim.

- Non-dictatorship: obvious.

The Gibbard-Satterthwaite theorem seems to quash any hope of designing incentive compatible social choice functions. The whole field of Mechanism Design attempts escaping from this impossibility result using various modifications in the model. The next section describes how the addition of "money" offers an escape route. Chapter ?? offers other escape routes that do not rely on money.

\subsection{Mechanisms With Money}

In the previous section, we modeled a voter's preference as an order on the alternatives. $a \succ_{i} b$ implies that $i$ prefers $a$ to $b$, but we did not model "by how much" is $a$ preferred to $b$. "Money" is a yardstick that allows measuring this. Moreover, money can be transferred between players. The existence of money with these properties is an assumption, but a fairly reasonable one in many circumstances, and will allow us to do things that we could not do otherwise.

Formally, in this section we re-define our setting. We will still have a set of alternatives $A$ and a set of $n$ players $I$ (which we will no longer call voters). The preference of a player $i$ is now given by a valuation function $v_{i}: A \rightarrow \Re$, where $v_{i}(a)$ denotes the "value" that $i$ assigns to alternative $a$ being chosen. This value is in terms of some currency, i.e. we assume that if $a$ is chosen and then player $i$ is additionally given some quantity $m$ of money, then $i$ 's utility is $u_{i}=v_{i}(a)+m$, this utility being the abstraction of what the player desires and aims to maximize. Utilities of this form are called quasilinear preferences, denoting the separable and linear dependence on money. 


\subsubsection{Vickrey's second price auction}

Before we proceed to the general setting, in this subsection we study a basic example: a simple auction. Consider a single item that is auctioned for sale among $n$ players. Each player $i$ has a scalar value $w_{i}$ that he is "willing to pay" for this item. More specifically, if he wins the item, but has to pay some price $p$ for it, then his utility is $w_{i}-p$, while if someone else wins the item then $i$ 's utility is 0 . Putting this scenario into the terms of our general setting, the set of alternatives here is the set of possible winners, $A=\{i$-wins $\mid i \in I\}$, and the valuation of each bidder $i$ is $v_{i}(i$-wins $)=w_{i}$ and $v_{i}(j$-wins $)=0$ for all $j \neq i$. A natural social choice would be to allocate the item to the player who values it highest: choose $i$-wins where $i=\operatorname{argmax}_{j} w_{j}$. However, the challenge is that we do not know the values $w_{i}$ but rather each player knows his own value, and we want to make sure that our mechanism decides on the allocation - the social choice - in a way that cannot be strategically manipulated. Our degree of freedom is the definition of the payment by the winner.

Let us first consider the two most natural choices of payment and see why they do not work as intended:

- No payment: In this version we give the item for free to the player with highest $w_{i}$. Clearly, this method is easily manipulated: every player will benefit by exaggerating his $w_{i}$, reporting a much larger $w_{i}^{\prime}>>w_{i}$ that can cause him to win the item, even though his real $w_{i}$ is not the highest.

- Pay your bid: An attempt of correction will be to have the winner pay the declared bid. However, this system is also open to manipulation: a player with value $w_{i}$ who wins and pays $w_{i}$ gets a total utility of 0 . Thus it is clear that he should attempt declaring a somewhat lower value $w_{i}^{\prime}<w_{i}$ that still wins. In this case he can still win the item getting a value of $w_{i}$ (his real value) but paying only the smaller $w_{i}^{\prime}$ (his declared value), obtaining a net positive utility $u_{i}=w_{i}-w_{i}^{\prime}>0$. What value $w_{i}^{\prime}$ should $i$ bid then? Well, if $i$ knows the value of the second highest bid, then he should declare just above it. But what if he does not know?

Here is the solution:

Definition 1.12 Vickrey's second price auction: Let the winner be the player $i$ with the highest declared value of $w_{i}$, and let $i$ pay the second highest declared bid $p^{*}=\max _{j \neq i} w_{j}$.

Now it turns out that manipulation never can increase any players' utility. Formally 
Proposition 1.13 (Vickrey): For every $w_{1} \ldots w_{n}$ and every $w_{i}^{\prime}$, Let $u_{i}$ be $i$ 's utility if he bids $w_{i}$ and $u_{i}^{\prime}$ his utility if he bids $w_{i}^{\prime}$. Then, $u_{i} \geq u_{i}^{\prime}$.

Proof Assume that by saying $w_{i}$ he wins, and that the second highest (reported) value is $p^{*}$, then $u_{i}=w_{i}-p^{*} \geq 0$. Now, for an attempted manipulation $w_{i}^{\prime}>p^{*}, i$ would still win if he bids $w_{i}^{\prime}$ and would still pay $p^{*}$, thus $u_{i}^{\prime}=u_{i}$. On the other hand for $w_{i}^{\prime} \leq p^{*}, i$ would lose so $u_{i}^{\prime}=0 \leq u_{i}$.

If $i$ loses by bidding $w_{i}$, then $u_{i}=0$. Let $j$ be the winner in this case, and thus $w_{j} \geq w_{i}$. For $w_{i}^{\prime}<w_{j}, i$ would still lose and so $u_{i}^{\prime}=0=u_{i}$. For $w_{i}^{\prime} \geq w_{j}, i$ would win, but would pay $w_{j}$, thus his utility would be $u_{i}^{\prime}=w_{i}-w_{j} \leq 0=u_{i}$.

This very simple and elegant idea achieves something that is quite remarkable: it reliably computes a function (argmax) of $n$ numbers (the $w_{i}$ 's) that are each held secretly by a different self-interested player! Taking a philosophical point of view, this may be seen as the mechanics for the implementation of Adam Smith's invisible hand: despite private information and pure selfish behavior, social welfare is achieved. All the field of Mechanism Design is just a generalization of this possibility.

\subsubsection{Incentive Compatible Mechanisms}

In a world with money our mechanisms will not only choose a social alternative but will also determine monetary payments to be made by the different players. The complete social choice is then composed of the alternative chosen as well as of the transfer of money. Never the less, we will refer to each of these part separately, calling the alternative chosen the social choice, not including in this term the monetary payments.

Formally, a mechanism needs to socially choose some alternative from $A$, as well as to decide on payments. The preference of each player $i$ is modeled by a valuation function $v_{i}: A \rightarrow \Re$, where $v_{i} \in V_{i}$. Throughout the rest of this chapter $V_{i} \subseteq \Re^{A}$ is a commonly known set of possible valuation functions for player $i$.

Starting at this point and for the rest of this chapter, it will be convenient to use the following standard notation:

Notation: Let $v=\left(v_{1} \ldots v_{n}\right)$ be an $n$-dimensional vector. We will denote the $(n-1)$-dimensional vector in which the $i$ 'th coordinate is removed by $v_{-i}=\left(v_{1} \ldots v_{i-1}, v_{i+1}, \ldots v_{n}\right)$. Thus we have three equivalent notations: $v=$ $\left(v_{1} \ldots v_{n}\right)=\left(v_{i}, v_{-i}\right)$. Similarly, for $V=V_{1} \times \ldots \times V_{n}$, we will denote $V_{-i}=$ $V_{1} \times \ldots \times V_{i-1} \times V_{i+1} \times \ldots \times V_{n}$. Similarly we will use $t_{-i}, x_{-i}, X_{-i}$, etc. 
Definition 1.14 A (direct revelation) mechanism is a social choice function $f: V_{1} \times \ldots \times V_{n} \rightarrow A$ and a vector of payment functions $p_{1} \ldots p_{n}$, where $p_{i}: V_{1} \times \ldots \times V_{n} \rightarrow \Re$ is the amount that player $i$ pays.

The qualification "direct revelation" will become clear in section 1.4 where we will generalize the notion of a mechanism further. We are now ready for the key definition in this area, incentive compatibility also called strategyproofness or truthfulness.

Definition 1.15 A mechanism $\left(f, p_{1} \ldots p_{n}\right)$ is called incentive compatible if for every player $i$, every $v_{1} \in V_{1}, \ldots, v_{n} \in V_{n}$ and every $v_{i}^{\prime} \in V_{i}$, if we denote $a=f\left(v_{i}, v_{-i}\right)$ and $a^{\prime}=f\left(v_{i}^{\prime}, v_{-i}\right)$, then $v_{i}(a)-p_{i}\left(v_{i}, v_{-i}\right) \geq v_{i}\left(a^{\prime}\right)-p_{i}\left(v_{i}^{\prime}, v_{-i}\right)$.

Intuitively this means that player $i$ whose valuation is $v_{i}$ would prefer "telling the truth" $v_{i}$ to the mechanism rather than any possible "lie" $v_{i}^{\prime}$, since this gives him higher (in the weak sense) utility.

\subsubsection{Vickrey-Clarke-Groves mechanisms}

While in the general setting without money, as we have seen, nothing nontrivial is incentive compatible, the main result in this setting is positive and provides an incentive compatible mechanism for the most natural social choice function: optimizing the social welfare. The social welfare of an alternative $a \in A$ is the sum of the valuations of all players for this alternative, $\sum_{i} v_{i}(a)$.

Definition 1.16 A mechanism $\left(f, p_{1} \ldots p_{n}\right)$ is called a Vickrey-Clarke-Groves (VCG) mechanism if:

- $f\left(v_{1} \ldots v_{n}\right) \in \operatorname{argmax}_{a \in A} \sum_{i} v_{i}(a)$. I.e. $f$ maximizes the social welfare.

- for some functions $h_{1} \ldots h_{n}$, where $h_{i}: V_{-i} \rightarrow \Re$ (i.e. $h_{i}$ does not depend on $\left.v_{i}\right)$, we have that for all $v_{1} \in V_{1}, \ldots, v_{n} \in V_{n}: p_{i}\left(v_{1} \ldots v_{n}\right)=h_{i}\left(v_{-i}\right)-$ $\sum_{j \neq i} v_{j}\left(f\left(v_{1} \ldots v_{n}\right)\right)$.

The main idea lies in the term $-\sum_{j \neq i} v_{j}\left(f\left(v_{1} \ldots v_{n}\right)\right)$ which means that each player is paid an amount equal to the sum of the values of all other players. When this term is added to his own value $v_{i}\left(f\left(v_{1} \ldots v_{n}\right)\right)$, the sum becomes exactly the total social welfare of $f\left(v_{1} \ldots v_{n}\right)$. Thus this mechanism aligns all players' incentives with the social goal of maximizing social welfare, which is exactly archived by telling the truth. The other term in the payment $h_{i}\left(v_{i}\right)$ has no strategic implications for player $i$ since it does not depend in any way on what he says, and thus from player $i$ 's point of view it is just 
a constant. Of course, the choice of $h_{i}$ does change significantly how much money is paid and in which direction, but we will postpone this discussion. What we have just intuitively explained is:

Theorem 1.17 (Vickrey-Clarke-Groves) Every VCG mechanism is incentive compatible.

Let us prove it formally

Proof Fix $i, v_{-i}, v_{i}$, and $v_{i}^{\prime}$. We need to show that for player $i$ with valuation $v_{i}$, the utility when declaring $v_{i}$ is not less than the utility when declaring $v_{i}^{\prime}$. Denote $a=f\left(v_{i}, v_{-i}\right)$ and $a^{\prime}=f\left(v_{i}^{\prime}, v_{-i}\right)$. The utility of $i$ when declaring $v_{i}$ is $v_{i}(a)+\sum_{j \neq i} v_{j}(a)-h_{i}\left(v_{-i}\right)$, but when declaring $v_{i}^{\prime}$ is $v_{i}\left(a^{\prime}\right)+\sum_{j \neq i} v_{j}\left(a^{\prime}\right)-h_{i}\left(v_{-i}\right)$. But since $a=f\left(v_{i}, v_{-i}\right)$ maximizes social welfare over all alternatives, $v_{i}(a)+\sum_{j \neq i} v_{j}(a) \geq v_{i}\left(a^{\prime}\right)+\sum_{j \neq i} v_{j}\left(a^{\prime}\right)$ and thus the same inequality holds when subtracting the same term $h_{i}\left(v_{-i}\right)$ from both sides.

\subsubsection{Clarke Pivot Rule}

Let us now return to the question of choosing the "right" $h_{i}$ 's. One possibility is certainly choosing $h_{i}=0$. This has the advantage of simplicity but usually does not make sense since the mechanism pays here a great amount of money to the players. Intuitively we would prefer that players pay money to the mechanism, but not more than the gain that they get. Here are two conditions that seem to make sense, at least in a setting where all valuations are non-negative:

Definition 1.18 - A mechanism is (ex-post) individually rational if players always get non-negative utility. Formally if for every $v_{1} \ldots v_{n}$ we have that $v_{i}\left(f\left(v_{1} \ldots v_{n}\right)\right)-p_{i}\left(v_{1} \ldots v_{n}\right) \geq 0$.

- A mechanism has no positive transfers if no player is ever paid money.

Formally if for every $v_{1} \ldots v_{n}$ and every $i, p_{i}\left(v_{1} \ldots v_{n}\right) \geq 0$.

The following choice of $h_{i}$ 's provides these two properties:

Definition 1.19 (Clarke pivot rule) The choice $h_{i}\left(v_{-i}\right)=\max _{b \in A} \sum_{j \neq i} v_{i}(b)$ is called the Clarke pivot payment. Under this rule the payment of player $i$ is $p_{i}\left(v_{1} \ldots v_{n}\right)=\max _{b} \sum_{j \neq i} v_{i}(b)-\sum_{j \neq i} v_{i}(a)$, where $a=f\left(v_{1} \ldots v_{n}\right)$.

Intuitively, $i$ pays an amount equal to the total damage that he causes the other players - the difference between the social welfare of the others 
with and without $i$ 's participation. In other words, the payments make each player internalize the externalities that he causes.

Lemma 1.20 A VCG mechanism with Clarke pivot payments makes no positive transfers. If $v_{i}(a) \geq 0$ for every $v_{i} \in V_{i}$ and $a \in A$ then it is also individually rational.

Proof Let $a=f\left(v_{1} \ldots v_{n}\right)$ be the alternative maximizing $\sum_{j} v_{j}(a)$ and $b$ be the alternative maximizing $\sum_{j \neq i} v_{j}(b)$. To show individual rationality, the utility of player $i$ is $v_{i}(a)+\sum_{j \neq i} v_{j}(a)-\sum_{j \neq i} v_{j}(b) \geq \sum_{j} v_{j}(a)-\sum_{j} v_{j}(b) \geq$ 0 , where the first inequality is since $v_{i}(b) \geq 0$ and the second is since $a$ was chosen as to maximize $\sum_{j} v_{j}(a)$. To show no positive transfers note that $p_{i}\left(v_{1} \ldots v_{n}\right)=\sum_{j \neq i} v_{i}(b)-\sum_{j \neq i} v_{i}(a) \geq 0$, since $b$ was chosen as to maximize $\sum_{j \neq i} v_{j}(b)$

As stated, the Clarke pivot rule does not fit many situations where valuations are negative, i.e. when alternatives have costs to the players. Indeed, with the Clarke pivot rule players always pay money to the mechanism, while the natural interpretation in case of costs would be the opposite. The spirit of the Clarke pivot rule in such cases can be captured by a modified rule that chooses $b$ as to maximize the social welfare "when $i$ does not participate" where the exact meaning of this turns out to be quite natural in most applications.

\subsubsection{Examples}

\subsubsection{Auction of a single item}

The Vickrey auction that we started our discussion with is a special case of a VCG mechanism with the Clarke pivot rule. Here $A=\{i$-wins $\mid i \in I\}$. Each player has value 0 if he does not get the item, and may have any positive value if he does win the item, thus $V_{i}=\left\{v_{i} \mid v_{i}(i\right.$-wins $) \geq 0$ and $\forall j \neq$ $i, v_{i}(j$-wins $\left.)=0\right\}$. Notice that finding the player with highest value is exactly equivalent to maximizing $\sum_{i} v_{i}(i)$ since only a single player gets non-zero value. VCG payments using the Clarke pivot rule give exactly Vickrey's second price auction.

\subsubsection{Reverse Auction}

In a reverse auction (procurement auction) the bidder wants to procure an item from the bidder with lowest cost. In this case the valuation spaces are given by $V_{i}=\left\{v_{i} \mid v_{i}(i\right.$-wins $) \leq 0$ and $\forall j \neq i v_{i}(j$-wins $\left.)=0\right\}$, and indeed 
procuring the item from the lowest cost bidder is equivalent to maximizing the social welfare. The natural VCG payment rule would be for the mechanism to pay to the lowest bidder an amount equal to the second lowest bid, and pay nothing to the others. This may be viewed as capturing the spirit of the pivot rule since the second lowest bid is what would happen "without i".

\subsubsection{Bilateral trade}

In the bilateral trade problem a seller holds an item and values it at some $0 \leq v_{s} \leq 1$ and a potential buyer values it at some $0 \leq v_{b} \leq 1$. (The constants 0 and 1 are arbitrary and may be replaced with any commonly known constants $0 \leq v_{l} \leq v_{h}$.) The possible outcomes are $A=\{$ no-trade,trade and social efficiency implies that trade is chosen if $v_{b}>v_{s}$ and no-trade if $v_{s}>v_{b}$. Using VCG payments and decreeing that no payments be made in case of no-trade, implies that in case of trade the buyer pays $v_{s}$ and the seller is paid $v_{b}$. Notice that since in this case $v_{b}>v_{s}$, the mechanism subsidizes the trade. As we will see below in section 1.5.5, this is unavoidable.

\subsubsection{Multi-unit auctions}

In a multi-unit auction, $k$ identical units of some good are sold in an auction (where $k<n$ ). In the simple case each bidder is interested in only a single unit. In this case $A=\{S$-wins $|S \subset I| S \mid,=k\}$, and a bidder's valuation $v_{i}$ gives some fixed value $v^{*}$ if $i$ gets an item, i.e. $v_{i}(S)=v^{*}$ if $i \in S$ and $v_{i}(S)=0$ otherwise. Maximizing social welfare means allocating the items to the $k$ highest bidders, and in the VCG mechanism with the pivot rule, each of them should pay the $k+1$ 'st highest offered price. (Losers pay 0.)

In a more general case bidders may be interested in more than a single unit and have a different value for each number of units obtained. The next level of sophistication comes when the items in the auction are heterogeneous, and valuations can give a different value to each combination of items. This is called a combinatorial auction and is studied at length in chapter ??

\subsubsection{Public Project}

The government is considering undertaking a public project (e.g. building a bridge). The project has a commonly known cost $C$, and is valued by each citizen $i$ at (a privately known) value $v_{i}$ (We usually think that $v_{i} \geq 0$, but allowing $v_{i}<0$, i.e. citizens that are hurt by the project is also covered.) Social efficiency means that the government will undertake this project iff $\sum_{i} v_{i}>C$. (This is not technically a sub case of our definition of maximizing the social welfare, since our definition did not assume any costs or values for 
the designer, but becomes so by adding an extra player "government" whose valuation space is the singleton valuation, giving cost $C$ to undertaking the project and 0 otherwise.) The VCG mechanism with the Clarke pivot rule means that a player $i$ with $v_{i} \geq 0$ will pay a non-zero amount only if he is pivotal: $\sum_{j \neq i} v_{j} \leq C$ but $\sum_{j} v_{j}>C$ in which case he will pay $p_{i}=C-\sum_{j \neq i} v_{j}$. (A player with $v_{i}<0$ will make a non-zero payment only if $\sum_{j \neq i} v_{j}>C$ but $\sum_{j} v_{j} \leq C$ in which case he will pay $p_{i}=\sum_{j \neq i} v_{j}-C$.) One may verify that $\sum_{i} p_{i}<C$ (unless $\sum_{i} v_{i}=C$ ), and thus the payments collected do not cover the project's costs. As we will see in section 1.5.5, this is unavoidable.

\subsubsection{Buying a path in a network}

Consider a communication network, modeled as a directed graph $G=(V, E)$, where each link $e \in E$ is owned by a different player, and has a cost $c_{e} \geq 0$ if his link is used for carrying some message. Suppose that we wish to procure a communication path between two specified vertices $s, t \in V$, i.e. the set of alternatives is the set of all possible $s-t$ paths in $G$, and player $e$ has value 0 if the path chosen does not contain $e$ and value $-c_{e}$ if the path chosen does contain $e$. Maximizing social welfare means finding the shortest path $p$ (in terms of $\left.\sum_{e \in p} c_{e}\right)$. A VCG mechanism, that makes no payments to edges that are not in $p$, will pay to each $e_{0} \in p$ the quantity $\sum_{e \in p^{\prime}} c_{e}-\sum_{e \in p-\left\{e_{0}\right\}} c_{e}$ where $p$ is the shortest $s-t$ path in $G$ and $p^{\prime}$ is the shortest $s-t$ path in $G$ that does not contain the edge $e$ (for simplicity assume that $G$ is 2-edge connected so such a $p^{\prime}$ always exists). This corresponds to the spirit of the pivot rule since "without $e$ " the mechanism can simply not use paths that contain $e$.

\subsection{Implementation in Dominant Strategies}

In this section our aim is to put the issue of incentive compatibility in a wider context. The mechanisms considered so far extract information from the different players by motivating them to "tell the truth". More generally, one may think of other, indirect, methods of extracting sufficient information from the participants. Perhaps one may devise some complex protocol that achieves the required social choice when players act strategically. This section will formalize these more general mechanisms, and the associated notions describing what happens when "players act strategically".

Deviating from the common treatment in economics, in this section we will describe a model that does not involve any distributional assumptions. Many of the classical results of Mechanism Design are captured in this framework, 
including most of the existing applications in computational settings. In section 1.6 we will add this ingredient of distributional assumptions reaching the general "Bayesian" models.

\subsubsection{Games with strict incomplete information}

How do we model strategic behavior of the players when they are missing some of the information that specifies the game? Specifically in our setting a player does not know the private information of the other players, information that determines their preferences. The standard setting in Game Theory supposes on the other hand that the "rules" of the game, including the utilities of all players, are public knowledge.

We will use a model of games with independent private values and strict incomplete information. Let us explain the terms: "independent private values" means that the utility of a player depends fully on his private information and not on any information of others as it is independent from his own information. Strict incomplete information is a (not completely standard) term that means that we will have no probabilistic information in the model. An alternative term sometimes used is "pre-Bayesian". From a CS perspective, it means that we will use a worst case analysis over unknown information. So here is the model:

Definition 1.21 A game with (independent private values and) strict incomplete information for a set of $n$ players is given by the following ingredients:

(i) For every player $i$, a set of actions $X_{i}$.

(ii) For every player $i$, a set of types $T_{i}$. A value $t_{i} \in T_{i}$ is the private information that $i$ has.

(iii) For every player $i$, a utility function $u_{i}: T_{i} \times X_{1} \times \ldots \times X_{n} \rightarrow \Re$, where $u_{i}\left(t_{i}, x_{1} \ldots x_{n}\right)$ is the utility achieved by player $i$, if his type (private information) is $t_{i}$, and the profile of actions taken by all players is $x_{1} \ldots x_{n}$.

The main idea that we wish to capture with this definition is that each player $i$ must choose his action $x_{i}$ when knowing $t_{i}$ but not the other $t_{j}$ 's. Note that the $t_{j}$ 's do not affect his utility, but they do affect how the other players behave. Thus the interplay between the different $x_{i}$ 's is more delicate than in "regular" games. The total behavior of player $i$ in such a setting is captured by a function that specifies which action $x_{i}$ is taken for every 
possible type $t_{i}$ - this is termed a strategy. It is these strategies that we want to be in equilibrium.

Definition 1.22 - A strategy of a player $i$ is a function $s_{i}: T_{i} \rightarrow X_{i}$.

- A profile of strategies $s_{1} \ldots s_{n}$ is an ex-post-Nash equilibrium if for every $t_{1} \ldots t_{n}$ we have that the actions $s_{1}\left(t_{1}\right) \ldots s_{n}\left(t_{n}\right)$ are in Nash equilibrium in the full information game defined by the $t_{i}$ 's. Formally: For all $i$, all $t_{1} \ldots t_{n}$, and all $x_{i}^{\prime}$ we have that $u_{i}\left(t_{i}, s_{i}\left(t_{i}\right), s_{-i}\left(t_{-i}\right)\right) \geq u_{i}\left(t_{i}, x_{i}^{\prime}, s_{-i}\left(t_{-i}\right)\right)$.

- A strategy $s_{i}$ is a (weakly) dominant strategy if for every $t_{i}$ we have that the action $s_{i}\left(t_{i}\right)$ is a dominant strategy in the full information game defined by $t_{i}$. Formally: for all $t_{i}$, all $x_{-i}$ and all $x_{i}^{\prime}$ we have that $u_{i}\left(t_{i}, s_{i}\left(t_{i}\right), x_{-i}\right) \geq u_{i}\left(t_{i}, x_{i}^{\prime}, x_{-i}\right)$. A profile $s_{1} \ldots s_{n}$ is called a dominant strategy equilibrium if each $s_{i}$ is a dominant strategy.

Thus the notion of ex-post Nash requires that $s_{i}\left(t_{i}\right)$ is a best response to $s_{i}\left(t_{-i}\right)$ for every possible value of $t_{-i}$, i.e. without knowing anything about $t_{-i}$ but rather only knowing the forms of the other players' strategies $s_{-i}$ as functions. The notion of dominant strategy requires that $s_{i}\left(t_{i}\right)$ is a best response to any $x_{-i}$ possible - i.e. without knowing anything about $t_{-i}$ or about $s_{-i}$. Both of these definitions seem too good to be true: how likely is it that a player has a single action that is a best response to all $x_{-i}$ or even to all $s_{-i}\left(t_{-i}\right)$ ? Indeed in usual cases one does not expect games with strict incomplete information to have any of these equilibria. However, in the context of Mechanism Design - where we get to design the game - we can sometimes make sure that they do exist.

While at first sight the notion of dominant strategy equilibrium seems much stronger than ex-post-Nash, this is only due to actions that are never used:

Proposition 1.23 Let $s_{1} \ldots s_{n}$ be an ex-post-Nash equilibrium of a game $\left(X_{1} \ldots X_{n} ; T_{1} \ldots T_{n} ; u_{1} \ldots u_{n}\right)$. Define $X_{i}^{\prime}=\left\{s_{i}\left(t_{i}\right) \mid t_{i} \in T_{i}\right\}$ (i.e. $X_{i}^{\prime}$ is the actual range of $s_{i}$ in $\left.X_{i}\right)$, then $s_{1} \ldots s_{n}$ is a dominant strategy equilibrium in the game $\left(X_{1}^{\prime} \ldots X_{n}^{\prime} ; T_{1} \ldots T_{n} ; u_{1} \ldots u_{n}\right)$.

Proof Let $x_{i}=s_{i}\left(t_{i}\right) \in X_{i}^{\prime}, x_{i}^{\prime} \in X_{i}^{\prime}$, and for every $j \neq i x_{j} \in X_{j}^{\prime}$. By definition of $X_{j}^{\prime}$, for every $j \neq i$, there exists $t_{j}^{\prime} \in T_{j}$ such that $s_{j}\left(t_{j}\right)=$ $x_{j}$. Since $s_{1} \ldots s_{n}$ is an ex-post-Nash equilibrium, $u_{i}\left(t_{i}, s_{i}\left(t_{i}\right), s_{-i}\left(t_{-i}\right)\right) \geq$ $u_{i}\left(t_{i}, x_{i}^{\prime}, s_{-i}\left(t_{-i}\right)\right)$, and as $x_{-i}=s_{-i}\left(t_{-i}\right)$ we get exactly $u_{i}\left(t_{i}, s_{i}\left(t_{i}\right), x_{-i}\right) \geq$ $u_{i}\left(t_{i}, x_{i}^{\prime}, x_{-i}\right)$ as required in the definition of dominant strategies. 


\subsubsection{Mechanisms}

We are now ready to formalize the notion of a general - non direct revelation - mechanism. The idea is that each player has some private information $t_{i} \in T_{i}$ that captures his preference over a set of alternatives $A$, i.e. $v_{i}\left(t_{i}, a\right)$ is the value that player $i$ assigns to $a$ when his private information is $t_{i}$. We wish to "implement" some social choice function $f: T_{1} \ldots T_{n} \rightarrow A$ that aggregates these preferences. We design a "mechanism" for this purpose: this will be some protocol for interaction with the players, specifying what each can "say" and what is done in each case. Formally, we can specify a set of possible actions $X_{i}$ for each player, an outcome function $a: X_{1} \times \ldots \times X_{n} \rightarrow$ $A$ that chooses an alternative in $A$ for each profile of actions, and payment functions $p: X_{1} \times \ldots \times X_{n} \rightarrow \Re$ that specify the payment of each player for every profile of actions. Now the players are put in a game with strict incomplete information and we may expect them to reach an equilibrium point (if such exists).

Definition 1.24 - A mechanism for $n$ players is given by (a) players' type spaces $T_{1} \ldots T_{n}$, (b) players' action spaces $X_{1} \ldots X_{n}$, (c) an alternative set $A$, (d) players' valuations functions $v_{i}: T_{i} \times A: \rightarrow \Re$, (e) an outcome function $a: X_{1} \times \ldots \times X_{n} \rightarrow A$, and (f) payment functions $p_{1} \ldots p_{n}$, where $p_{i}: X_{1} \times \ldots \times X_{n} \rightarrow \Re$. The game with strict incomplete information induced by the mechanism is given by using the types spaces $T_{i}$, the action spaces $X_{i}$, and the utilities $u_{i}\left(t_{i}, x_{1} \ldots x_{n}\right)=v_{i}\left(t_{i}, a\left(x_{1} \ldots x_{n}\right)\right)-p_{i}\left(x_{1} \ldots x_{n}\right)$.

- The mechanism implements a social choice function $f: T_{1} \times T_{n} \rightarrow A$ in dominant strategies if for some dominant strategy equilibrium $s_{1} \ldots s_{n}$ of the induced game, where $s_{i}: T_{i} \rightarrow X_{i}$, we have that for all $t_{1} \ldots t_{n}$, $f\left(t_{1} \ldots t_{n}\right)=a\left(s_{1}\left(t_{1}\right) \ldots s_{n}\left(t_{n}\right)\right)$.

- Similarly we say that the mechanism implements $f$ in ex-post-equilibrium if for some ex-post equilibrium $s_{1} \ldots s_{n}$ of the induced game we have that for all $t_{1} \ldots t_{n}, f\left(t_{1} \ldots t_{n}\right)=a\left(s_{1}\left(t_{1}\right) \ldots s_{n}\left(t_{n}\right)\right)$.

Clearly every dominant strategy implementation is also an ex-post Nash implementation. Note that our definition only requires that for some equilibrium $f\left(t_{1} \ldots t_{n}\right)=a\left(s_{1}\left(t_{1}\right) \ldots s_{n}\left(t_{n}\right)\right)$ and allows other equilibria to exist. A stronger requirement would be that all equilibria have this property, or stronger still, that only a unique equilibrium point exists. 


\subsubsection{The Revelation Principle}

At first sight it seems that the more general definition of mechanisms will allow us to do more than is possible using incentive compatible direct revelation mechanisms introduced in section 1.3. This turns out to be false: any general mechanism that implements a function in dominant strategies can be converted into an incentive compatible one.

Proposition 1.25 (Revelation principle) If there exists an arbitrary mechanism that implements $f$ in dominant strategies, then there exists an incentive compatible mechanism that implements $f$. The payments of the players in the incentive compatible mechanism are identical to those, obtained at equilibrium, of the original mechanism.

Proof The proof is very simple: the new mechanism will simply simulate the equilibrium strategies of the players. I.e. Let $s_{1} \ldots s_{n}$ be a dominant strategy equilibrium of the original mechanism, we define a new direct revelation mechanism: $f\left(t_{1} \ldots t_{n}\right)=a\left(s_{1}\left(t_{1}\right) \ldots s_{n}\left(t_{n}\right)\right)$ and $p_{i}^{\prime}\left(t_{1} \ldots t_{n}\right)=$ $p_{i}\left(s_{1}\left(t_{1}\right) \ldots s_{n}\left(t_{n}\right)\right)$. Now, since each $s_{i}$ is a dominant strategy for player $i$, then for every $t_{i}, x_{-i}, x_{i}^{\prime}$ we have that $v_{i}\left(t_{i}, a\left(s_{i}\left(t_{i}\right), x_{-i}\right)\right)-p_{i}\left(s_{i}\left(t_{i}\right), x_{-i}\right) \geq$ $v_{i}\left(t_{i}, a\left(x_{i}^{\prime}, x_{-i}\right)\right)-p_{i}\left(x_{i}^{\prime}, x_{-i}\right)$. Thus in particular this is true for all $x_{-i}=$ $s_{-i}\left(t_{-i}\right)$ and any $x_{i}^{\prime}=s_{i}\left(t_{i}^{\prime}\right)$, which gives the definition of incentive compatibility of the mechanism $\left(f, p_{1}^{\prime} \ldots p_{n}^{\prime}\right)$.

Corollary 1.26 If there exists an arbitrary mechanism that ex-post-Nash implements $f$, then there exists an incentive compatible mechanism that implements $f$. Moreover, the payments of the players in the incentive compatible mechanism are identical to those, obtained in equilibrium, of the original mechanism.

Proof We take the ex-post implementation and restrict the action space of each player, as in proposition 1.23, to those that are taken, for some input type, in the ex-post equilibrium $s_{1} \ldots s_{n}$. Proposition 1.23 states that now $s_{1} \ldots s_{n}$ is a dominant strategy equilibrium of the game with the restricted spaces, and thus the mechanism with the restricted action spaces is an implementation in dominant strategies. We can now invoke the revelation principle to get an incentive compatible mechanism.

The revelation principle does not mean that indirect mechanisms are useless. In particular, general mechanisms may be adaptive (multi-round), significantly reducing the communication (or computation) burden of the 
players or of the auctioneer relative to a non-adaptive direct mechanism. An example is the case of combinatorial auctions studied in chapter ??

\subsection{Characterizations of Incentive Compatible Mechanisms}

In section 1.3 we saw how to implement the most natural social choice function: maximization of the social welfare. The question that drives this section is: what other social choice functions can we implement? In economic settings, the main reasons for attempting implementations of other social choice functions are increasing the revenue or introducing some kind of fairness. In computerized settings there are many natural optimization goals and we would like to be able to implement each of them. For example in scheduling applications, a common optimization goal is that of the "makespan" - completion time of the last job. This is certainly a social choice function that is very different than maximizing the total social welfare - how can it be implemented? Another major motivation for social choice functions that do not maximize social welfare comes from computational considerations. In many applications the set of alternatives $A$ is complex, and maximizing social welfare is a hard computational problem (NP-complete). In many of these cases there are computationally efficient algorithms that approximate the maximum social welfare. Such an algorithm in effect gives a social choice function that approximates social welfare maximization, but is different from it. Can it be implemented?

Chapter ?? and parts of chapter ?? address these issues specifically. This section limits itself to laying the foundations by providing basic characterizations of implementable social choice functions and their associated payments.

Due to the revelation principle, we can restrict ourselves again to look at incentive compatible mechanisms. Thus, in this section we revert to the notation used in subsection 1.3.3: A mechanism $M=\left(f, p_{1} \ldots p_{n}\right)$ over domain of preferences $V_{1} \times \ldots \times V_{n}\left(V_{i} \subseteq \Re^{A}\right)$ is composed of a social choice function $f: V_{1} \times \ldots \times V_{n} \rightarrow A$ and payment functions $p_{1} \ldots p_{n}$, where $p_{i}$ : $V_{1} \times \ldots \times V_{n} \rightarrow \Re$ is the amount that player $i$ pays. In the rest of the section we will provide characterizations of when such mechanisms are incentive compatible.

\subsubsection{Direct Characterization}

We start by stating explicitly the required properties from an incentive compatible mechanism. 
Proposition 1.27 A mechanism is incentive compatible if and only if it satisfies the following conditions for every $i$ and every $v_{-i}$ :

(i) The payment $p_{i}$ does not depend on $v_{i}$, but only on the alternative chosen $f\left(v_{i}, v_{-i}\right)$. I.e., for every $v_{-i}$, there exist prices $p_{a} \in \Re$, for every $a \in A$, such that for all $v_{i}$ with $f\left(v_{i}, v_{-i}\right)=a$ we have that $p\left(v_{i}, v_{-i}\right)=p_{a}$.

(ii) The mechanism optimizes for each player. I.e. for every $v_{i}$, we have that $f\left(v_{i}, v_{-i}\right) \in \operatorname{argmax}_{a}\left(v_{i}(a)-p_{a}\right)$, where the quantification is over all alternatives in the range of $f\left(\cdot, v_{-i}\right)$.

Proof (if part) Denote $a=f\left(v_{i}, v_{-i}\right), a^{\prime}=f\left(v_{i}^{\prime}, v_{-i}\right), p_{a}=p\left(v_{i}, v_{-i}\right)$, and $p_{a^{\prime}}=p\left(v_{i}^{\prime}, v_{-i}\right)$. The utility of $i$ when telling the truth is $v_{i}(a)-p_{a}$, which is not less than the utility when declaring $v_{i}^{\prime}, v_{i}\left(a^{\prime}\right)-p_{a^{\prime}}$, since the mechanism optimizes for $i$, i.e. $a=f\left(v_{i}, v_{-i}\right) \in \operatorname{argmax}_{a}\left(v_{i}(a)-p_{a}\right)$.

(only-if part; first condition) If for some $v_{i}, v_{i}^{\prime}, f\left(v_{i}, v_{-i}\right)=f\left(v_{i}^{\prime}, v_{-i}\right)$ but $p_{i}\left(v_{i}, v_{-i}\right)>p_{i}\left(v_{i}^{\prime}, v_{-i}\right)$ then a player with type $v_{i}$ will increase his utility by declaring $v_{i}^{\prime}$.

(only-if part; second condition) If $f\left(v_{i}, v_{-i}\right) \notin \operatorname{argmax}_{a}\left(v_{i}(a)-p_{a}\right)$, fix $a^{\prime} \in \operatorname{argmax}_{a}\left(v_{i}(a)-p_{a}\right)$ in the range of $f\left(\cdot, v_{-i}\right)$, and thus for some $v_{i}^{\prime}, a^{\prime}=$ $f\left(v_{i}^{\prime}, v_{-i}\right)$. Now a player with type $v_{i}$ will increase his utility by declaring $v_{i}^{\prime}$.

\subsubsection{Weak Monotonicity}

The previous characterization involves both the social choice function and the payment functions. We now provide a partial characterization that only involves the social choice function. In section 1.5.5 we will see that the social choice function usually determines the payments essentially uniquely.

Definition 1.28 A social choice function $f$ satisfies Weak Monotonicity (WMON) if for all $i$, all $v_{-i}$ we have that $f\left(v_{i}, v_{-i}\right)=a \neq b=f\left(v_{i}^{\prime}, v_{-i}\right)$ implies that $v_{i}(a)-v_{i}(b) \geq v_{i}^{\prime}(a)-v_{i}^{\prime}(b)$.

I.e. WMON means that if the social choice changes when a single player changes his valuation, then it must be because the player increased his value of the new choice relative to his value of the old choice.

Theorem 1.29 If a mechanism $\left(f, p_{1} \ldots p_{n}\right)$ is incentive compatible then $f$ satisfies WMON. If all domains of preferences $V_{i}$ are convex sets (as subsets of an Euclidean space) then for every social choice function that satisfies 
WMON there exists payment functions $p_{1} \ldots p_{n}$ such that $\left(f, p_{1} \ldots p_{n}\right)$ is incentive compatible.

The first part of the theorem is easy and we will bring it completely, the second part is quite involved, and will not be given here. It is known that WMON is not a sufficient condition for incentive compatibility in general non-convex (more precisely, non-simply-connected) domains.

Proof (First part) Assume first that $\left(f, p_{1} \ldots p_{n}\right)$ is incentive compatible, and fix $i$ and $v_{-i}$ in an arbitrary manner. Proposition 1.27 implies the existence of fixed prices $p_{a}$ for all $a \in A$ (that do not depend on $v_{i}$ ) such that whenever the outcome is $a$ then bidder $i$ pays exactly $p_{a}$. Now assume $f\left(v_{i}, v_{-i}\right)=a \neq$ $b=f\left(v_{i}^{\prime}, v_{-i}\right)$. Since a player with valuation $v_{i}$ does not prefer declaring $v_{i}^{\prime}$ we have that $v_{i}(a)-p_{a} \geq v_{i}(b)-p_{b}$. Similarly since a player with valuation $v_{i}^{\prime}$ does not prefer declaring $v_{i}$ we have that $v_{i}^{\prime}(a)-p_{a} \leq v_{i}^{\prime}(b)-p_{b}$. Subtracting the second inequality from the first we get $v_{i}(a)-v_{i}(b) \geq v_{i}^{\prime}(a)-v_{i}^{\prime}(b)$, as required.

While WMON gives a pretty tight characterization of implementable social choice functions, it still leaves something to be desired as it is not intuitively clear what exactly are the WMON functions. The problem is that the WMON condition is a local condition for each player separately and for each $v_{-i}$ separately. Is there a global characterization? This turns out to depend intimately on the domains of preferences $V_{i}$. For two extreme cases there are good global characterizations: when $V_{i}$ is "unrestricted" i.e. $V_{i}=\Re^{A}$, and when $V_{i}$ is severely restricted as to be essentially single dimensional. These two cases are treated in the next two subsections below. The intermediate range where the $V_{i}$ 's are somewhat restricted, a range in which most computationally interesting problems lie, is still wide open. More on this appears in chapter ??.

\subsubsection{Weighted VCG}

It turns out that when the domain of preferences is unrestricted, then the only incentive compatible mechanisms are simple variations of the VCG mechanism. These variations allow giving weights to the players, weights to the alternatives, and allow restricting the range. The resulting social choice function is an "affine maximizer":

Definition 1.30 A social choice function $f$ is called an affine maximizer if 
for some subrange $A^{\prime} \subset A$, for some player weights $w_{1} \ldots w_{n} \in \Re^{+}$and for some outcome weights $c_{a} \in \Re$ for every $a \in A^{\prime}$, we have that $f\left(v_{1} \ldots v_{n}\right) \in$ $\operatorname{argmax}_{a \in A^{\prime}}\left(c_{a}+\sum_{i} w_{i} v_{i}(a)\right)$.

It is easy to see that VCG mechanisms can be generalized to affine maximizers:

Proposition 1.31 Let $f$ be an affine maximizer. Define for every $i, p_{i}\left(v_{1} \ldots v_{n}\right)=$ $h_{i}\left(v_{-i}\right)-\sum_{j \neq i}\left(w_{j} / w_{i}\right) v_{j}(a)-c_{a} / w_{i}$, where $h_{i}$ is an arbitrary function that does not depend on $v_{i}$. Then, $\left(f, p_{1} . . p_{n}\right)$ is incentive compatible.

Proof First, we can assume wlog that $h_{i}=0$. The utility of player $i$ if alternative $a$ is chosen is $v_{i}(a)+\sum_{j \neq i}\left(w_{j} / w_{i}\right) v_{j}(a)+c_{a} / w_{i}$. By multiplying by $w_{i}>0$, this expression is maximized when $c_{a}+\sum_{j} w_{j} v_{j}(a)$ is maximized which is what happens when $i$ reports $v_{i}$ truthfully.

Roberts' theorem states that for unrestricted domains with at least 3 possible outcomes, these are the only incentive compatible mechanisms.

Theorem 1.32 (Roberts) If $|A| \geq 3, f$ is onto $A, V_{i}=\Re^{A}$ for every $i$, and $\left(f, p_{1} \ldots p_{n}\right)$ is incentive compatible then $f$ is an affine maximizer.

The proof of this theorem is not trivial and is given in chapter ??. It is easy to see that the restriction $|A| \geq 3$ is crucial (as in Arrow's theorem), since the case $|A|=2$ falls into the category of "single parameter" domains discussed below, for which there do exist incentive compatible mechanisms beyond weighted VCG. It remains open to what extent can the restriction of $V_{i}=\Re^{A}$ be relaxed.

\subsubsection{Single-parameter domains}

The unrestricted case $V_{i}=\Re^{A}$ basically means that the valuation space has full dimensionality. The opposite case is when the space $V_{i}$ is singledimensional, i.e. there is a single real parameter that directly determines the whole vector $v_{i}$. There are several possible levels of generality in which to formalize this, and we will consider one of intermediate generality which is simple and yet suffices for most applications. In our setting each bidder has a private scalar value for "winning", with "losing" having value of 0 . This is modeled by some commonly-known subset of winning alternatives $W_{i} \subseteq A$. The main point is that all winning alternatives are equivalent to each other for player $i$; and similarly all losing outcomes are equivalent to each other. All the examples in section 1.3.5 fall into this category. A simple 
example is an auction of one item where $W_{i}$ is the single outcome where $i$ wins. A more complex example is the setting of buying a path in a network (subsection 1.3.5.6), where $W_{i}$ is the set of all paths that contain edge $i$.

Definition 1.33 A single parameter domain $V_{i}$ is defined by a (publicly known) $W_{i} \subset A$ and a range of values $\left[t^{0}, t^{1}\right] . V_{i}$ is the set of $v_{i}$ such that for some $t^{0} \leq t \leq t^{1}, v_{i}(a)=t$ for all $a \in W_{i}$ and $v_{i}(a)=0$ for all $a \notin W_{i}$. In such settings we will abuse notation and use $v_{i}$ as the scalar $t$.

For this setting it is quite easy to completely characterize incentive compatible mechanisms.

Definition 1.34 A social choice function $f$ on a single parameter domain is called monotone in $v_{i}$ if for every $v_{-i}$ and every $v_{i} \leq v_{i}^{\prime} \in \Re$ we have that $f\left(v_{i}, v_{-i}\right) \in W_{i}$ implies that $f\left(v_{i}^{\prime}, v_{-i}\right) \in W_{i}$. I.e. if valuation $v_{i}$ makes $i$ win, then so will every higher valuation $v_{i}^{\prime} \geq v_{i}$.

For a monotone function $f$, for every $v_{-i}$ for which player $i$ can both win and lose, there is always a critical value below which $i$ loses and above which he wins. E.g. in a second price auction the critical value for each player is highest declared value among the other players.

Definition 1.35 The critical value of a monotone social choice function $f$ on a single parameter domain is $c_{i}\left(v_{-i}\right)=\sup _{v_{i}: f\left(v_{i}, v_{-i}\right) \notin W_{i}} v_{i}$. The critical value at $v_{-i}$ is undefined if $\left\{v_{i} \mid f\left(v_{i}, v_{-i}\right) \notin W_{i}\right\}$ is empty.

We will call a mechanism on a single parameter domain "normalized" if the payment for losing is always 0 , i.e. for every $v_{i}, v_{-i}$ such that $f\left(v_{i}, v_{-i}\right) \notin W_{i}$ we have that $p_{i}\left(v_{i}, v_{-i}\right)=0$. It is not difficult to see that every incentive compatible mechanism may be easily turned into a normalized one, so it suffices to characterize normalized mechanisms.

Theorem 1.36 A normalized mechanism $\left(f, p_{1} \ldots p_{n}\right)$ on a single parameter domain is incentive compatible if and only if the following conditions hold:

(i) $f$ is monotone in every $v_{i}$.

(ii) Every winning bid pays the critical value. (Recall that losing bids pay 0.) Formally, For every $i, v_{i}, v_{-i}$ such that $f\left(v_{i}, v_{-i}\right) \in W_{i}$, we have that $p_{i}\left(v_{i}, v_{-i}\right)=c_{i}\left(v_{-i}\right)$. (If $c_{i}\left(v_{-i}\right)$ is undefined we require instead that for every $v_{-i}$, there exists some value $c_{i}$, such that $p_{i}\left(v_{i}, v_{-i}\right)=c_{i}$ for all $v_{i}$ such that $f\left(v_{i}, v_{-i}\right) \in W_{i}$.) 
Proof (If part) Fix $i, v_{-i}, v_{i}$. For every declaration made by $i$, if he wins his utility is $v_{i}-c_{i}\left(v_{-i}\right)$ and if he loses his utility is 0 . Thus he prefers winning if $v_{i}>c_{i}\left(v_{-i}\right)$ and losing if $v_{i}<c_{i}\left(v_{-i}\right)$, which is exactly what happens when he declares the truth.

(Only-if part, first condition) If $f$ is not monotone then for some $v_{i}^{\prime}>v_{i}$ we have that $f\left(v_{i}^{\prime}, v_{-i}\right)$ loses while $f\left(v_{i}, v_{-i}\right)$ wins and pays some amount $p=p_{i}\left(v_{i}, v_{-i}\right)$. Since a bidder with value $v_{i}$ is not better off bidding $v_{i}^{\prime}$ and losing we have that $v_{i}-p \geq 0$. Since a bidder with value $v_{i}^{\prime}$ is not better off bidding $v_{i}$ and winning we have that $v_{i}^{\prime}-p \leq 0$. Contradiction.

(Only-if part, second condition) Assume that some winning $v_{i}$ pays $p>$ $c_{i}\left(v_{-i}\right)$ then, using proposition 1.27, all winning bids will make the same payment, including a winning $v_{i}^{\prime}$ with $c_{i}\left(v_{-i}\right)<v_{i}^{\prime}<p$. But such a bidder is better off losing which he can do by bidding some value $v^{\text {lose }}<c\left(v_{-i}\right)$. In the other direction if $v_{i}$ pays $p<c\left(v_{-i}\right)$ then a losing $v_{i}^{\prime}$ with $c\left(v_{-i}\right)>v_{i}^{\prime}>p$ is better of wining and paying $p$, which will happen if he bids $v_{i}$.

Notice that this characterization leaves ample space for non-affine-maximization. For example we can implement social functions such as maximizing the euclidean norm $\operatorname{argmax}_{a} \sum_{i} v_{i}(a)^{2}$ or maximizing the minimum value $\operatorname{argmax}_{a} \min _{i} v_{i}(a)$. Indeed in many cases this flexibility allows the design of computationally efficient approximation mechanisms for problems whose exact optimization is computationally intractable - an example is given in chapter ??.

\subsubsection{Uniqueness of prices}

This section has so far focused on characterizing the implementable social choice functions. What about the payment functions? It turns out that the payment function is essentially uniquely determined by the social choice function. "Essentially" means that if we take an incentive compatible mechanisms with payments $p_{i}$ and modify the payments to $p_{i}^{\prime}\left(v_{1} \ldots v_{n}\right)=$ $p_{i}\left(v_{1} \ldots v_{n}\right)+h_{i}\left(v_{-i}\right)$ for an arbitrary function $h_{i}$ that does not depend on $v_{i}$, then incentive compatibility remains. It turns out that this is the only leeway in the payment:

Theorem 1.37 Assume that the domains of preference $V_{i}$ are connected sets in the usual metric in the Euclidean space. Let $\left(f, p_{1} \ldots p_{n}\right)$ be an incentive compatible mechanism. The mechanism with modified payments $\left(f, p_{1}^{\prime} \ldots p_{n}^{\prime}\right)$ is incentive compatible if and only if for some functions $h_{i}$ : $V_{-i} \rightarrow \Re$ we have that $p_{i}^{\prime}\left(v_{1} \ldots v_{n}\right)=p_{i}\left(v_{1} \ldots v_{n}\right)+h_{i}\left(v_{-i}\right)$ for all $v_{1} \ldots v_{n}$. 
Proof The "if" part is clear since $h_{i}$ has no strategic implications for player $i$, so we need only prove the only-if part. Assume that $\left(f, p_{1}^{\prime} \ldots p_{n}^{\prime}\right)$ is incentive compatible, and for the rest of the proof fix some $i$ and some $v_{-i}$.

For every $a \in A$ denote $V^{a}=\left\{v_{i} \in V_{i} \mid f\left(v_{i}, v_{-i}\right)=a\right\}$. Using proposition 1.27 , the payment $p\left(v_{i}, v_{-i}\right)$ is identical for all $v_{i} \in V^{a}$ and will be denoted by $p_{a}$. Similarly we denote $p_{a}^{\prime}=p^{\prime}\left(v_{i}, v_{-i}\right)$ for some $v_{i} \in V^{a}$. It now suffices to show that for every $a, b \in A, p_{a}-p_{b}=p_{a}^{\prime}-p_{b}^{\prime}$.

For $a, b \in A$ we will say that $a$ and $b$ are close if for every $\epsilon>0$ there exist $v_{i}^{a}, v_{i}^{b} \in V_{i}$ such that $\left\|v_{i}^{a}-v_{i}^{b}\right\|=\max _{c \in A}\left|v_{i}^{a}(c)-v_{i}^{b}(c)\right| \leq \epsilon$, and $f\left(v_{i}^{a}, v_{-i}\right)=$ $a$ and $f\left(v_{i}^{b}, v_{-i}\right)=b$. We will first prove the required $p_{a}-p_{b}=p_{a}^{\prime}-p_{b}^{\prime}$ for close $a, b$. Fix $v_{i}^{a}, v_{i}^{b} \in V_{i}$ as in the definition of closeness. Since a bidder with type $v_{i}^{a}$ does not gain by declaring $v_{i}^{b}$ with payments $p$, we have that $v_{i}^{a}(a)-p_{a} \geq v_{i}^{a}(b)-p_{b}$, and since a bidder with $v_{i}^{b}$ does not gain by declaring $v_{i}^{a}$ we have that $v_{i}^{b}(a)-p_{a} \leq v_{i}^{b}(b)-p_{b}$. Putting together and rearranging we have that $v_{i}^{a}(b)-v_{i}^{a}(a) \leq p_{b}-p_{a} \leq v_{i}^{b}(b)-v_{i}^{b}(a)$. Similarly, by considering the mechanism with payments $p^{\prime}$ we have $v_{i}^{a}(b)-v_{i}^{a}(a) \leq p_{b}^{\prime}-p_{a}^{\prime} \leq v_{i}^{b}(b)-v_{i}^{b}(a)$. But now recall that $\left\|v_{i}^{a}-v_{i}^{b}\right\| \leq \epsilon$ and thus the upper bound and the lower bound for $p_{b}-p_{a}$ and for $p_{b}^{\prime}-p_{a}^{\prime}$ are at most $2 \epsilon$ apart and thus $\left|\left(p_{b}-p_{a}\right)-\left(p_{b}^{\prime}-p_{a}^{\prime}\right)\right| \leq 2 \epsilon$. Since $\epsilon$ was arbitrary $p_{b}-p_{a}=p_{b}^{\prime}-p_{a}^{\prime}$.

To show $p_{b}-p_{a}=p_{b}^{\prime}-p_{a}^{\prime}$ for general (not necessarily close) $a$ and $b$, consider $B=\left\{b \in A \mid p_{b}-p_{a}=p_{b}^{\prime}-p_{a}^{\prime}\right\}$. Since $p_{b}-p_{a}=p_{b}^{\prime}-p_{a}^{\prime}$ and $p_{c}-p_{b}=p_{c}^{\prime}-p_{b}^{\prime}$ implies $p_{c}-p_{a}=p_{c}^{\prime}-p_{a}^{\prime}$ we have that no alternative in $A-B$ can be close to any alternative in $B$. Thus $V^{B}=\bigcup_{b \in B} V^{b}$ has positive distance from its complement $V^{A-B}=\bigcup_{b \notin B} V^{b}$ contradicting the connectedness of $V$.

It is not difficult to see that the assumption that $V_{i}$ is connected is essential, as for example, if the valuations are restricted to be integral, then modifying $p_{i}$ by any small constants $\epsilon<1 / 2$ will not modify incentive compatibility.

From this, and using the revelation principle, we can directly get many corollaries:

(i) The only incentive compatible mechanisms that maximize social welfare are those with VCG payments.

(ii) In the bilateral trade problem (section 1.3.5.3) the only incentive compatible mechanism which maximizes social welfare and makes no payments in case of no-trade is the one shown there which subsidizes the trade. More generally, if a mechanism for bilateral trade satisfies ex-post individual rationality, then it cannot dictate positive pay- 
ments from the players in case of no-trade and thus it must subsidize trade.

(iii) In the public project problem (section 1.3.5.5) no ex-post individually rational mechanism that maximizes social welfare can recover the cost of the project. Again, the uniqueness of payments imply that if players with value 0 pay 0 (which is as much as they can pay maintaining individual rationality) then their payments in case of building the project must be identical to those obtained using the Clarke pivot rule.

In section 1.6.3 we will see a similar theorem in the Bayesian setting, a theorem that will strengthen all of these corollaries as well to that setting.

\subsubsection{Randomized Mechanisms}

All of our discussion so far considered only deterministic mechanisms. It is quite natural to allow also randomized mechanisms. Such mechanisms would be allowed to produce a distribution over alternatives and a distribution over payments. Alternatively, but specifying slightly more structure, we can allow distributions over deterministic mechanisms. This will allow us to distinguish between two notions of incentive compatibility.

Definition 1.38 - A randomized mechanism is a distribution over deterministic mechanisms (all with the same players, types spaces $V_{i}$, and outcome space $A$.)

- A randomized mechanism is incentive compatible in the universal sense if every deterministic mechanism in the support is incentive compatible.

- A randomized mechanism is incentive compatible in expectation if truth is a dominant strategy in the game induced by expectation. I.e. if for all $i$, all $v_{i}, v_{-i}$, and $v_{i}^{\prime}$, we have that $E\left[v_{i}(a)-p_{i}\right] \geq E\left[v_{i}\left(a^{\prime}\right)-p_{i}^{\prime}\right]$, where $\left(a, p_{i}\right)$, and $\left(a^{\prime}, p_{i}^{\prime}\right)$ are random variables denoting the outcome and payment when $i$ bids, respectively, $v_{i}$ and $v_{i}^{\prime}$, and $E[\cdot]$ denotes expectation over the randomization of the mechanism.

It is clear that incentive compatibility in the universal sense implies incentive compatibility in expectation. For most purposes incentive compatibility in expectation seems to be the more natural requirement. The universal definition is important if players are not risk neutral (which we do not consider in this chapter) or if the mechanism's internal randomization is not completely hidden from the players. As we will see in chapters ?? and ?? randomized mechanisms can often be useful and achieve more than deterministic ones. 
We will now characterize randomized incentive compatible mechanisms over single parameter domains. Recall the single parameter setting and notations from section 1.5.4. We will denote the probability that $i$ wins by $w_{i}\left(v_{i}, v_{-i}\right)=\operatorname{Pr}\left[f\left(v_{i}, v_{-i}\right) \in W_{i}\right]$ (probability taken over the randomization of the mechanism) and will use $p_{i}\left(v_{i}, v_{-i}\right)$ to directly denote the expected payment of $i$. In this notation the utility of player $i$ with valuation $v_{i}$ when declaring $v_{i}^{\prime}$ is $v_{i} \cdot w\left(v_{i}^{\prime}, v_{-i}\right)-p_{i}\left(v_{i}^{\prime}, v_{-i}\right)$. For ease of notation we will focus on normalized mechanisms in which the lowest bid $v_{i}^{0}=t^{0}$ loses completely $w_{i}\left(v_{i}^{0}, v_{-i}\right)=0$ and pays nothing $p_{i}\left(v_{i}^{0}, v_{-i}\right)=0$.

Theorem 1.39 A normalized randomized mechanism in a single parameter domain is incentive compatible in expectation if and only if for every $i$ and every fixed $v_{-i}$ we have that

(i) The function $w_{i}\left(v_{i}, v_{-i}\right)$ is monotonically non decreasing in $v_{i}$.

(ii) $p_{i}\left(v_{i}, v_{-i}\right)=v_{i} \cdot w\left(v_{i}, v_{-i}\right)-\int_{v_{i}^{0}}^{v_{i}} w\left(t, v_{-i}\right) d t$.

Proof In the proof we will simplify notation by removing the index $i$ and the fixed argument $v_{-i}$ everywhere. In this notation, to show incentive compatibility we need to establish that $v w(v)-p(v) \geq v w\left(v^{\prime}\right)-p\left(v^{\prime}\right)$ for every $v^{\prime}$. Plugging in the formula for $p$ we get $\int_{v^{0}}^{v} w(t) d t \geq \int_{v^{0}}^{v^{\prime}} w(t) d t-\left(v^{\prime}-\right.$ $v) w\left(v^{\prime}\right)$. For $v^{\prime}>v$ this is equivalent to $\left(v^{\prime}-v\right) w\left(v^{\prime}\right) \geq \int_{v}^{v^{\prime}} w(t) d t$ which is true due to the monotonicity of $w$. For $v^{\prime}<v$ we get $\left(v-v^{\prime}\right) w\left(v^{\prime}\right) \leq$ $\int_{v^{\prime}}^{v} w(t) d t$ which again is true due to the monotonicity of $w$.

In the other direction, combining the incentive constraint at $v, v w(v)-$ $p(v) \geq v w\left(v^{\prime}\right)-p\left(v^{\prime}\right)$, with the incentive constraint at $v^{\prime}, v^{\prime} w(v)-p(v) \leq$ $v^{\prime} w\left(v^{\prime}\right)-p\left(v^{\prime}\right)$, and subtracting the inequalities, we get $\left(v^{\prime}-v\right) w(v) \leq$ $\left(v^{\prime}-v\right) w\left(v^{\prime}\right)$ which implies monotonicity of $w$.

To derive the formula for $p$, we can re-arrange the two incentive constraints as

$$
v \cdot\left(w\left(v^{\prime}\right)-w(v)\right) \leq p\left(v^{\prime}\right)-p(v) \leq v^{\prime} \cdot\left(w\left(v^{\prime}\right)-w(v)\right) .
$$

Now by letting $v^{\prime}=v+\epsilon$, dividing throughout by $\epsilon$, and taking the limit, both sides approach the same value, $v \cdot d w / d v$, and we get $d p / d v=v \cdot d w / d v$. Thus, taking into account the normalization condition $p\left(v^{0}\right)=0$, we have that $p\left(v_{i}\right)=\int_{v^{0}}^{v_{i}} v \cdot w^{\prime}(v) d v$, and integrating by parts completes the proof. (This seems to require the differentiability of $w$, but as $w$ is monotone this holds almost everywhere, which suffices since we immediately integrate.) 
We should point out explicitly that the randomization in a randomized mechanism is completely controlled by the mechanism designer and has nothing to do with any distributional assumptions on players' valuations as will be discussed in the next section.

\subsection{Bayesian-Nash Implementation}

So far in this chapter we have only considered implementation in dominant strategies (and the very similar ex-post-Nash). As mentioned in section 1.4 this is usually considered too strict a definition in economic theory. It models situations where each player has no information at all about the private information of the others - not even a prior distribution - and must operate under a "worst case" assumption. The usual working definition in economic theory takes a Bayesian approach, assumes some commonly known prior distribution, and assumes that a player that lacks some information will optimize in a Bayesian sense according to the information that he does have. The formalization of these notions, mostly by Harsanyi, was a major development in economic theory in the 1960s and 1970s, and is certainly still the dominant approach to handling lack of information in economic theory. In this section we will give these basic notions in the context of mechanism design, again limiting ourselves to settings with independent private values.

\subsubsection{Bayesian-Nash Equilibrium}

Definition 1.40 A game with (independent private values and) incomplete information on a set of $n$ players is given by the following ingredients:

(i) For every player $i$, a set of actions $X_{i}$.

(ii) For every player $i$, a set of types $T_{i}$, and a prior distribution $D_{i}$ on $T_{i}$. A value $t_{i} \in T_{i}$ is the private information that $i$ has, and $D_{i}\left(t_{i}\right)$ is the a priori probability that $i$ gets type $t_{i}$.

(iii) For every player $i$, a utility function $u_{i}: T_{i} \times X_{1} \times \ldots \times X_{n} \rightarrow \Re$, where $u_{i}\left(t_{i}, x_{1} \ldots x_{n}\right)$ is the utility achieved by player $i$, if his type (private information) is $t_{i}$, and the profile of actions taken by all players is $x_{1} \ldots x_{n}$.

The main idea that we wish to capture with this definition is that each player $i$ must choose his action $x_{i}$ when knowing $t_{i}$ but not the other $t_{j}$ 's but rather only knowing the prior distribution $D_{j}$ on each other $t_{j}$. The behavior of player $i$ in such a setting is captured by a function that specifies 
which action $x_{i}$ is taken for every possible type $t_{i}$ - this is termed a strategy. It is these strategies that we would want to be in equilibrium.

Definition 1.41 A strategy of a player $i$ is a function $s_{i}: T_{i} \rightarrow X_{i}$. A profile of strategies $s_{1} \ldots s_{n}$ is a Bayesian-Nash equilibrium if for every player $i$ and every $t_{i}$ we have that $s_{i}\left(t_{i}\right)$ is the best response that $i$ has to $s_{-i}()$ when his type is $t_{i}$, in expectation over the types of the other players. Formally: For all $i$, all $t_{i}$, and all $x_{i}^{\prime}: E_{D_{-i}}\left[u_{i}\left(t_{i}, s_{i}\left(t_{i}\right), s_{-i}\left(t_{-i}\right)\right)\right] \geq E_{D_{-i}}\left[u_{i}\left(t_{i}, x_{i}^{\prime}, s_{-i}\left(t_{-i}\right)\right)\right]$ (where $E_{D_{-i}}[]$ denotes the expectation over the other types $t_{-i}$ being chosen according to distribution $D_{-i}$ ).

This now allows us to define implementation in the Bayesian sense:

Definition 1.42 A Bayesian mechanism for $n$ players is given by (a) players' type spaces $T_{1} \ldots T_{n}$ and prior distributions on them $D_{1} \ldots D_{n}$, (b) players' action spaces $X_{1} \ldots X_{n}$, (c) an alternative set $A$, (d) players' valuations functions $v_{i}: T_{i} \times A: \rightarrow \Re,(\mathrm{e})$ an outcome function $a: X_{1} \times \ldots \times X_{n} \rightarrow A$, and (f) payment functions $p_{1} \ldots p_{n}$, where $p_{i}: X_{1} \times \ldots \times X_{n} \rightarrow \Re$.

The game with incomplete information induced by the mechanism is given by using the type spaces $T_{i}$ with prior distributions $D_{i}$, the action spaces $X_{i}$, and the utilities $u_{i}\left(t_{i}, x_{1} \ldots x_{n}\right)=v_{i}\left(t_{i}, a\left(x_{1} \ldots x_{n}\right)\right)-p_{i}\left(x_{1} \ldots x_{n}\right)$.

The mechanism implements a social choice function $f: T_{1} \times \ldots \times T_{n} \rightarrow$ $A$ in the Bayesian sense if for some Bayesian-Nash equilibrium $s_{1} \ldots s_{n}$ of the induced game $\left(s_{i}: T_{i} \rightarrow X_{i}\right)$ we have that for all $t_{1} \ldots t_{n}, f\left(t_{1} \ldots t_{n}\right)=$ $a\left(s_{1}\left(t_{1}\right) \ldots s_{n}\left(t_{n}\right)\right)$.

In particular it should be clear that every ex-post-Nash implementation is by definition also a Bayesian implementation for any distributions $D_{i}$. In general, however, being a Bayesian implementation depends on the distributions $D_{i}$ and there are many cases where a Bayesian-Nash equilibrium exists even though no dominant-strategy one does. A simple example - a first price auction - is shown in the next subsection. Just like in the case of dominant-strategy implementations, Bayesian implementations can also be turned into ones that are truthful in a Bayesian sense.

Definition 1.43 A mechanism is truthful in the Bayesian sense if (a) it is "direct revelation" i.e. the type spaces are equal to the action spaces $T_{i}=X_{i}$, and (b) the truthful strategies $s_{i}\left(t_{i}\right)=t_{i}$ are a Bayesian-Nash equilibrium.

Proposition 1.44 (Revelation principle) If there exists an arbitrary mechanism that implements $f$ in the Bayesian sense, then there exists a truthful 
mechanism that implements $f$ in the Bayesian sense. Moreover, the expected payments of the players in the truthful mechanism are identical to those, obtained in equilibrium, in the original mechanism.

The proof is similar to the proof of the same principle in the dominantstrategy setting given in proposition 1.25.

\subsubsection{First price auction}

As an example of Bayesian analysis we study the standard first price auction in a simple setting: a single item is auctioned between two players, Alice and Bob. Each has a private value for the item: $a$ is Alice's value and $b$ is Bob's value. While we already saw that a second price auction will allocate the item to the one with higher value, here we ask what would happen if the auction rules are the usual first-price ones: the highest bidder pays his bid. Certainly Alice will not bid $a$ since if she does even if she wins her utility will be 0 . She will thus need to bid some $x<a$, but how much lower? If she knew that Bob would bid $y$, she would certainly bid $x=y+\epsilon$ (as long as $x \leq a$ ). But she does not know $y$ or even $b$ which $y$ would depend on she only knows the distribution $D_{B o b}$ over $b$.

Let us now see how this situation falls in the Bayesian-Nash setting described above: The type space $T_{\text {Alice }}$ of Alice and $T_{B o b}$ of Bob is the non-negative real numbers, with $t_{\text {Alice }}$ denoted by $a$ and $t_{B o b}$ denoted by $b$. The distributions over the type space are $D_{\text {Alice }}$ and $D_{B o b}$. The action spaces $X_{\text {Alice }}$ and $X_{B o b}$ are also the non-negative real numbers, with $x_{\text {Alice }}$ denoted by $x$ and $x_{B o b}$ denoted by $y$. The possible outcomes are \{Alice-wins, Bob-wins $\}$, with $v_{\text {Alice }}($ Bob-wins $)=0$ and $v_{\text {Alice }}($ Alice-wins $)=$ $a$ (and similarly for Bob). The outcome function is that Alice-wins if $x \geq y$ and Bob-wins otherwise (we arbitrarily assume here that ties are broken in favor of Alice). Finally, the payment functions are $p_{\text {Alice }}=0$ whenever Bob-wins and $p_{\text {Alice }}=x$ whenever Alice-wins, while $p_{B o b}=y$ whenever Bob-wins and $p_{B o b}=0$ whenever Alice-wins. Our question translates into finding the Bayesian-Nash equilibrium of this game? Specifically we wish to find a strategy $s_{\text {Alice }}$ for Alice, given by a function $x(a)$, and a strategy $s_{B o b}$ for Bob, given by the function $y(b)$, that are in Bayesian equilibrium, i.e. are best-replies to each other.

In general, finding Bayesian-Nash equilibria is not an easy thing. Even for the this very simple first price auction the answer is not clear for general distributions $D_{\text {Alice }}$ and $D_{B o b}$. However, for the symmetric case where $D_{\text {Alice }}=D_{B o b}$, the situation is simpler and a closed form expression for 
the equilibrium strategies may be found. We will prove it for the special case of uniform distributions on the interval $[0,1]$. Similar arguments work for arbitrary non-atomic distributions over the valuations as well as for any number of bidders.

Lemma 1.45 In a first price auction among two players with prior distributions of the private values a, $b$ uniform over the interval $[0,1]$, the strategies $x(a)=a / 2$ and $y(b)=b / 2$ are in Bayesian-Nash equilibrium.

Note that in particular $x<y$ if and only if $a<b$ thus the winner is also the player with highest private value. This means that the first price auction also maximizes social welfare, just like a second-price auction.

Proof Let us consider which bid $x$ is Alice's optimal response to Bob's strategy $y=b / 2$, when Alice has value $a$. The utility for Alice is 0 if she loses and $a-x$ if she wins and pays $x$, thus her expected utility from bid $x$ is given by $u_{\text {Alice }}=\operatorname{Pr}[$ Alice wins with bid $x] \cdot(a-x)$, where the probability is over the prior distribution over $b$. Now Alice wins if $x \geq y$, and given Bob's strategy $y=b / 2$, this is exactly when $x \geq b / 2$. Since $b$ is distributed uniformly in $[0,1]$ we can readily calculate this probability: $2 x$ for $0 \leq x \leq 1 / 2,1$ for $x \geq 1 / 2$, and 0 for $x \leq 0$. It is easy to verify that the optimal value of $x$ is indeed in the range $0 \leq x \leq 1 / 2$ (since $x=1 / 2$ is clearly better than any $x>1 / 2$, and since any $x<0$ will give utility 0 ). Thus to optimize the value of $x$ we need to find the maximum of the function $2 x(a-x)$ over the range $0 \leq x \leq 1 / 2$. The maximum may be found by taking the derivative with respect to $x$ and equating it to 0 , which gives $2 a-4 x=0$, whose solution is $x=a / 2$ as required.

\subsubsection{Revenue Equivalence}

Let us now attempt comparing the first price auction and the second price auction. The social choice function implemented is exactly the same: giving the item to the player with highest private value. How about the payments? Where does the auctioneer get higher revenue? One can readily express the revenue of the second-price auction as $\min (a, b)$ and the revenue of the firstprice auction as $\max (a / 2, b / 2)$, and it is clear that each of these expressions is higher for certain values of $a$ and $b$.

But which is better on the average - in expectation over the prior distributions of $a$ and $b$ ? Simple calculations will reveal that the expected value of $\min (a, b)$ when $a$ and $b$ are chosen uniformly in $[0,1]$ is exactly $1 / 3$. Similarly the expected value of $\max (a / 2, b / 2)$ when $a$ and $b$ are chosen uniformly 
in $[0,1]$ is also exactly $1 / 3$. Thus both auctions generate equivalent revenue in expectation! This is no coincidence. It turns out that in quite general circumstances every two Bayesian-Nash implementations of the same social choice function generate the same expected revenue.

Theorem 1.46 (The Revenue Equivalence Principle): Under certain weak assumptions (to be detailed in the proof body), for every two BayesianNash implementations of the same social choice function $f$, we have that if for some type $t_{i}^{0}$ of player $i$, the expected (over the types of the other players) payment of player $i$ is the same in the two mechanisms, then it is the same for every value of $t_{i}$. In particular, if for each player $i$ there exists a type $t_{i}^{0}$ where the two mechanisms have the same expected payment for player $i$, then the two mechanisms have the same expected payments from each player and their expected revenues are the same.

Thus, for example, all single item auctions that allocate (in equilibrium) the item to the player with highest value and in which losers pay 0 , will have identical expected revenue.

The similarity to theorem 1.37 should be noted: in both cases it is shown that the allocation rule determines the payments, up to a normalization. In the case of dominant strategy implementation, this is true for every fixed type of the other players, while in the case of Bayesian-Nash implementation, this is true in expectation over that types of the others. The proofs of the two theorems look quite different due to technical reasons. The underlying idea is the same: take two "close" types, then the equations specifying that for neither type does a player gain by mis-representing himself as the other type, put together, determine the difference in payments in terms of the social choice function.

Proof Using the revelation principle, we can first limit ourselves to mechanisms that are truthful in the Bayesian-Nash sense. Let us denote by $V_{i}$ the space of valuation functions $v_{i}\left(t_{i}, \cdot\right)$ over all $t_{i}$.

Assumption 1: Each $V_{i}$ is convex. (Note that this holds for essentially every example we had so far. This condition can be replaced by pathconnectedness, and the proof becomes just slightly messier.)

Take any type $t_{i}^{1} \in T_{i}$. We will derive a formula for the expected payment for this type that depends only on the expected payment for type $t_{i}^{0}$ and on the social choice function $f$. Thus any two mechanisms that implement the same social choice function and have identical expected payments at $t_{i}^{0}$ will 
also have identical expected payments at $t_{i}^{1}$. For this, let us now introduce some notations:

- $v^{0}$ is the valuation $v\left(t_{i}^{0}, \cdot\right) \cdot v^{1}$ is the valuation $v\left(t_{i}^{1}, \cdot\right)$. We will look at these as vectors (in $V_{i} \subseteq \Re^{A}$ ), and look at their convex combinations $v^{\lambda}=v^{0}+\lambda\left(v_{1}-v_{0}\right)$. The convexity of $V_{i}$ implies that $v^{\lambda} \in V_{i}$ and thus there exists some type $t_{i}^{\lambda}$ such that $v^{\lambda}=v\left(t_{i}^{\lambda}, \cdot\right)$.

- $p^{\lambda}$ is the expected payment of player $i$ at type $t_{i}^{\lambda}: p^{\lambda}=E_{t_{-i}} p_{i}\left(t_{i}, t_{-i}\right)$.

- $w^{\lambda}$ is the probability distribution of $f\left(t_{i}^{\lambda}, \cdot\right)$, i.e. for every $a \in A$ $w^{\lambda}(a)=\operatorname{Pr}_{t_{-i}}\left[f\left(t_{i}^{\lambda}, t_{-i}\right)=a\right]$.

Assumption 2: $w^{\lambda}$ is continuously differentiable in $\lambda$. (This assumption is not really needed, but allows us to simply take derivatives and integrals as convenient.)

Once we have this notation in place, the proof is easy. Note that under these notations the expected utility of player $i$ with type $t_{i}^{\lambda}$ that declares $t_{i}^{\lambda^{\prime}}$ is given by the expression $v^{\lambda} \cdot w^{\lambda^{\prime}}-p^{\lambda^{\prime}}$. Since a player with type $t_{i}^{\lambda}$ prefers reporting the truth rather than $t_{i}^{\lambda+\epsilon}$ we have that $v^{\lambda} \cdot w^{\lambda}-p^{\lambda} \geq$ $v^{\lambda} \cdot w^{\lambda+\epsilon}-p^{\lambda+\epsilon}$. Similarly, a player with type $t_{i}^{\lambda+\epsilon}$ prefers reporting the truth rather than $t_{i}^{\lambda}$, so we have $v^{\lambda+\epsilon} \cdot w^{\lambda}-p^{\lambda} \leq v^{\lambda+\epsilon} \cdot w^{\lambda+\epsilon}-p^{\lambda+\epsilon}$. Rearranging and putting together, we get

$$
v^{\lambda}\left(w^{\lambda+\epsilon}-w^{\lambda}\right) \leq p^{\lambda+\epsilon}-p^{\lambda} \leq v^{\lambda+\epsilon}\left(w^{\lambda+\epsilon}-w^{\lambda}\right)
$$

Now divide throughout by $\epsilon$ and let $\epsilon$ approach 0 . $v^{\lambda+\epsilon}$ approaches $v^{\lambda}$, $\left(w^{\lambda+\epsilon}-w^{\lambda}\right) / \epsilon$ approaches the vector $d w^{\lambda} / d \lambda=w^{\prime}(\lambda)$ and thus we get that $\left.\left(p^{\lambda+\epsilon}-p^{\lambda}\right) / \epsilon\right)$ approaches $v^{\lambda} \cdot w^{\prime}(\lambda)$, and thus the derivative of $p^{\lambda}$ is defined and is continuous. Integrating, we get $p^{1}=p^{0}+\int_{0}^{1} v^{\lambda} \cdot w^{\prime}(\lambda) d \lambda$.

Thus the revenue equivalence theorem tells us that we cannot increase revenue without changing appropriately the allocation rule (social choice function) itself. In particular, all the corollaries in section 1.5.5 apply, in the sense of expectation, to all Bayesian-Nash implementations. However, if we are willing to modify the social choice function, then we can certainly increase revenue. Here is an example for the case of an auction with two bidders with valuations distributed uniformly in $[0,1]$ : Put a reservation price of $1 / 2$, and then sell to the highest bidder for a price that is the maximum of the low bid and the reservation price, $1 / 2$. If both bidders bid below the reservation price, then none of them wins. First, it is easy to verify that this rule is incentive compatible. Then a quick calculation will reveal that the expected revenue of this auction is $5 / 12$ which is more than 
the $1 / 3$ obtained by the regular second price or first price auctions. Chapter ?? discusses revenue maximization further.

\subsection{Further models}

This chapter has concentrated on basic models. Here we shortly mention several model extensions which address issues ignored by the basic models and have received attention in economic theory.

\subsubsection{Risk Aversion}

All of our discussion in the Bayesian model assumed that players are riskneutral: obtaining a utility of 2 with probability $1 / 2$ is equivalent to obtaining a utility of 1 with probability 1 . This is why we could just compute players' utilities by taking expectation. In reality, players are often riskaverse, preferring a somewhat lower utilities if they are more certain. A significant body of work in economic theory deals with formalizing and analyzing strategic behavior of such players. In our context, a particularly interesting observation is that the revenue equivalence principle fails and that with risk-averse bidders different mechanisms that implement the same social choice function may have different revenue. As an example it is known that first price auctions generate more revenue than second price auctions if the bidders are risk-averse.

\subsubsection{Interdependent Values}

We have only considered independent private value models: the types of the players are chosen independently of each other and each players' valuation depends only on his own private information. In a completely general setting, there would be some joint distribution over "states of the world" where such a state determines the valuations of all players. Players would not necessarily get as private information their own valuation, but rather each would get some "signal" - partial information about the state of the world - that provide some information about his own valuation and some about the valuations of others. Most of the results in this chapter cease holding for general models with interdependent values.

A case that is in the extreme opposite to the private value model is the "common value" model. In an auction of a single item under this model, we assume that the object in question has exactly the same value for all bidders. The problem is that none of them knows exactly what this value is 
and each players' signal only provides some partial information. An example is an auction for financial instruments such as bonds. Their exact value is not completely known as it depends on future interest rates, the probability of default, etc. What is clear though, is that whatever value the bonds will turn out to have, it will be the same for everyone. In such settings, an auction really serves as an information aggregation vehicle, reaching a joint estimate of the value by combining all players' signals. A common pitfall in such cases is the "winner's curse": if each bidder bids their own estimate of the object's common value, as determined from their own signal, then the winner will likely regret winning - the fact that a certain bidder won means that other signals implied a lower value, which likely means that the real value is lower than the estimate of the winner. Thus in equilibrium bidders must bid an estimate that is also conditioned on the fact that they win.

A commonly considered formalization that takes into account both a private value component and a common value component is that of affiliated signals. Roughly speaking, in such models each player gets a signal that is positively correlated (in a strong technical sense called affiliation) not only with his own value but also with the values of other players. In such settings, ascending English auctions are "better" (generate more revenue) than the non-adaptive second price auction (which is equivalent to an English auction in private value models): as the bidding progresses, each bidder gets information from the other bidders that increases his estimate of his value.

\subsubsection{Complete information models}

Our main point of view was that each player has its own private information. Some models consider a situation where all players have complete information about the game; it is only the mechanism designer who is lacking such information. A prototypical instance is that of King Solomon: two women, each claiming that the baby is hers. The women both know who the real mother is, but not King Solomon - he must design a mechanism that elicits this information from their different preferences. Several notions of implementation in such setting exists, and in general, mechanism design is much easier in this setting. In particular many implementations without money are possible.

\subsubsection{Hidden Actions}

All of the theory of Mechanism Design attempts overcoming the problem that players have private information that is not known to the mechanism 
designer. In many settings a different stumbling block occurs: players may perform hidden actions that are not visible to the "mechanism". This complementary difficulty to the private information difficulty has been widely studied in economics and has recently started to be considered in computer science settings.

\subsection{Notes}

Most of the material in this chapter can be found in graduate textbooks on micro-economics such as [MCWG95]. The books [Kri02, Kle04] on Auction theory contain more detail. As the Internet gained influence, during the 1990's, researchers in AI, Computer networks, and economics started noticing that Mechanism Design can be applied in computational settings. This was put forward in a general way in [NR01] who also coined the term Algorithmic Mechanism Design.

The earliest work on voting methods including that of Condorcet and Borda goes back to the late 18th century, appropriately around the time of the French Revolution. The modern treatment of social choice theory originates with the seminal work of [Arr51] where Arrow's theorem also appears. Over the years many proofs for Arrow's theorem have been put forward; we bring one of those in [Gea05]. The Gibbard-Satterthwaite theorem is due to [Gib73, Sat75]. The computational difficulty of manipulation of voting rules was first studied in [BTT89].

The positive results in Mechanism Design in the quasi-linear setting originate with the seminal work of [Vic61] who in particular studied single item auctions and multi-unit auctions with downward sloping valuations. The public project problem problem was studied in [Cla71] who also suggested the pivot rule, and the general formulation of what is now called VCG mechanisms appears in [Gro73]. The Bilateral Trade problem was studied in [MS83], and the application of buying a path in a network was put forward in [NR01].

The general framework of Mechanism Design and its basic notions have evolved in micro-economic theory mostly in the 1970s, and mostly in the general Bayesian setting which we only get to in section 1.6. Among the Influential papers in laying out the foundations are [Vic61, Cla71, Gro73, Sat75, GL77, DHM79, Mye81].

Early papers in algorithmic Mechanism Design, such as [NR01, LOS02], pointed out the necessity and difficulty of implementing social choice functions other than welfare maximization, due to other optimization goals or due to computational hardness. Characterizations of incentive compatible 
mechanisms have been previously obtained in economic theory as intermediate steps on the way to theorems with clear economic motivation. The discussion here tries to put it all together independently of particular intended applications. The weak monotonicity condition is from $\left[\mathrm{BCL}^{+} 06\right]$ and the sufficiency of this condition in convex domains is from [SY05]. The affine-maximization characterization in complete domains is from [Rob79], and [LMN03] attempts generalization to other domains. The uniqueness of pricing is the analog of the revenue equivalence theorem in the Bayesian setting which is due to [Mye81]; [GL77] showed it in the dominant strategy setting for welfare maximizing social choice functions. The corollary of the impossibility of budget-balanced bilateral trade appears in [MS83] in the Bayesian setting.

The Bayesian setting is currently the main vehicle of addressing lack of information in economic theory, and this development has mostly happened during the 1960s, with the main influence being the seminal work of [Har68]. As mentioned previously, most of development of the field of Mechanism Design noted above was in this setting. The revenue equivalence theorem, the form of the expected payment in single-parameter domains, as well as an analysis of revenue-maximizing auctions is from [Mye81].

Risk-averse bidders in (reverse) auctions are analyzed by [Hol80]. Auctions in the common value model are analyzed in [Wil77, Mil81]. The general model of interdependent valuations with affiliated signals was studied in [MW82]. Mechanism Design in complete information models is discussed in [Mas85, MR88].

\section{Acknowledgements}

I thank Shahar Dobzinski, Dana Fisman, Jason Hartline, Orna Kupferman, Ron Lavi, Ariel Procaccia and James Schummer for comments on earlier drafts of this chapter.

\section{Bibliography}

[Arr51] K. Arrow. Social Choice and Individual Values. Yale University Press, 1951. $\left[\mathrm{BCL}^{+} 06\right]$ S. Bikhchandani, S. Chatterji, R. Lavi, A. Mu'alem, N. Nisan, and A. Sen. Weak monotonicity characterizes deterministic dominant strategy implementation. Econometrica, 74(4), 2006.

[BTT89] J. Bartholdi, III, C. Tovey, and M. Trick. Computational difficulty of manipulating an election. Social Choice and Welfare, 6(3):227-241, 1989.

[Cla71] E. H. Clarke. Multipart pricing of public goods. Public Choice, pages 17-33, 1971.

[DHM79] P. Dasgupta, P. Hammond, and E. Maskin. The implementation of social 
choice rules: Some general results on incentive compatibility. The Review of Economic Studies, (46):185 - 216, 1979.

[Gea05] J. Geanakopolos. Three brief proofs of arrow's impossibility theorem. Economic Theory, 26(1):211 - 215, 2005.

[Gib73] Allan Gibbard. Manipulation of voting schemes: a general result. Econometrica, 41:587-601, 1973.

[GL77] J. Green and J.J. Laffont. Characterization of satisfactory mechanism for the revelation of preferences for public goods. Econometrica, pages 427-438, 1977.

[Gro73] T. Groves. Incentives in teams. Econometrica, pages 617-631, 1973.

[Har68] J. C. Harsanyi. Games with incomplete information played by 'bayesian' players, parts i ii and iii. Management science, 14, 1967-68.

[Hol80] C. Holt. Competitive bidding for contracts under alternative auction procedures. Journal of Political Economy, 88:433 - 445, 1980.

[Kle04] P. Klemperer. Auctions: Theory and Practice. Princeton University Press, 2004.

[Kri02] V. Krishna. Auction theory. Academic press, 2002.

[LMN03] R. Lavi, A. Mu'alem, and N. Nisan. Towrads a characterization of truthful combinatorial auctions. In FOCS, 2003.

[LOS02] Daniel Lehmann, Liadan Ita O'Callaghan, and Yoav Shoham. Truth revelation in approximately efficient combinatorial auctions. In $J A C M 49(5)$, pages 577-602, Sept. 2002.

[Mas85] E. Maskin. The theory of implementation in nash equilibrium. In Social Goals and Social organization: Essays in Honor of Elisha Pazner, 1985.

[MCWG95] A. Mas-Collel, W. Whinston, and J. Green. Microeconomic Theory. Oxford university press, 1995.

[Mil81] P. Milgrom. Rational expectations, information acquisition, and competitive bidding. Econometrica, 49:921 - 943, 1981.

[MR88] J. Moore and R. Repullo. Subgame perfect implementation. Econometrica, 56:1191 - 1220, 1988

[MS83] R. B. Myerson and M. Satterthwaite. Efficient mechanisms for bilateral trading. Journal of Economic Theory, (28):265 - 281, 1983.

[MW82] Paul R Milgrom and Robert J Weber. A theory of auctions and competitive bidding. Econometrica, 50(5):1089-1122, 1982.

[Mye81] R. B. Myerson. Optimal auction design. Mathematics of Operations Research, 6(1):58-73, 1981.

[NR01] Noam Nisan and Amir Ronen. Algorithmic mechanism design. Games and Economic Behaviour, 35:166 - 196, 2001.

[Rob79] K. Roberts. The characterization of implementable choice rules. In Aggregation and Revelation of Preferences, J-J. Laffont (ed.), North Holland Publishing Company., 1979.

[Sat75] Mark Allen Satterthwaite. Strategy-proofness and arrow's condition: Existence and correspondence theorems for voting procedures and social welfare functions. Journal of Economic Theory, pages 187-217, 1975.

[SY05] M. Saks and L. Yu. Weak monotonicity suffices for truthfulness. In EC, 2005 .

[Vic61] W. Vickrey. Counterspeculation, auctions and competitive sealed tenders. Journal of Finance, pages 8-37, 1961.

[Wil77] R. Wilson. A bidding model of perfect competition. Review of Economic Studies, 44:511 - 518, 1977. 